\title{
Evidence for light-by-light scattering in heavy-ion collisions with the ATLAS detector at the LHC
}

\author{
ATLAS Collaboration ${ }^{\dagger}$
}

\begin{abstract}
Light-by-light scattering $(\gamma \gamma \rightarrow \gamma \gamma)$ is a quantum-mechanical process that is forbidden in the classical theory of electrodynamics. This reaction is accessible at the Large Hadron Collider thanks to the large electromagnetic field strengths generated by ultra-relativistic colliding lead ions. Using $480 \mathrm{\mu b}^{-1}$ of lead-lead collision data recorded at a centre-of-mass energy per nucleon pair of $5.02 \mathrm{TeV}$ by the ATLAS detector, here we report evidence for light-by-light scattering. A total of 13 candidate events were observed with an expected background of $2.6 \pm 0.7$ events. After background subtraction and analysis corrections, the fiducial cross-section of the process $\mathrm{Pb}+\mathrm{Pb}(\gamma \gamma) \rightarrow \mathbf{P b}^{(*)}+\mathbf{P b}^{(*)} \gamma \gamma$, for photon transverse energy $E_{\mathrm{T}}>3 \mathrm{GeV}_{\text {, }}$ photon absolute pseudorapidity $|\eta|<2.4$, diphoton invariant mass greater than $6 \mathrm{GeV}$, diphoton transverse momentum lower than $2 \mathrm{GeV}$ and diphoton acoplanarity below 0.01 , is measured to be $70 \pm 24$ (stat.) \pm 17 (syst.) nb, which is in agreement with the standard model predictions.
\end{abstract}

O ne of the key features of Maxwell's equations is their linearity in both the sources and the fields, from which follows the superposition principle. This forbids effects such as light-by-light (LbyL) scattering, $\gamma \gamma \rightarrow \gamma \gamma$, which is a purely quantum-mechanical process. It was realized in the early history of quantum electrodynamics (QED) that LbyL scattering is related to the polarization of the vacuum ${ }^{1}$. In the standard model of particle physics, the virtual particles that mediate the LbyL coupling are electrically charged fermions or $W^{ \pm}$bosons. In QED, the $\gamma \gamma \rightarrow \gamma \gamma$ reaction proceeds at lowest order in the fine-structure constant $\left(\alpha_{\mathrm{em}}\right)$ via virtual one-loop box diagrams involving fermions (Fig. 1a), which is an $\mathcal{O}\left(\alpha_{\mathrm{em}}^{4} \approx 3 \times 10^{9}\right)$ process, making it challenging to test experimentally. Indeed, the elastic LbyL scattering has remained unobserved: even the ultra-intense laser experiments are not yet powerful enough to probe this phenomenon ${ }^{2}$.

LbyL scattering via an electron loop has been precisely, albeit indirectly, tested in measurements of the anomalous magnetic moment of the electron and muon ${ }^{3,4}$ where it is predicted to contribute substantially, as one of the QED corrections ${ }^{5}$. The $\gamma \gamma \rightarrow \gamma \gamma$ reaction has been measured in photon scattering in the Coulomb field of a nucleus (Delbrück scattering) at fixed photon energies below $7 \mathrm{GeV}$ (refs 6-9). The analogous process, where a photon splits into two photons by interaction with external fields (photon splitting), has been observed in the energy region of $0.1-0.5 \mathrm{GeV}$ (ref. 10). A related process involving only real photons, in which several photons fuse to form an electron-positron pair $\left(e^{+} e^{-}\right)$, has been measured in ref. 11. Similarly, the multiphoton Compton scattering, in which up to four laser photons interact with an electron, has been observed ${ }^{12}$.

An alternative way by which LbyL interactions can be studied is by using relativistic heavy-ion collisions. In 'ultra-peripheral collision' (UPC) events, with impact parameters larger than twice the radius of the nuclei ${ }^{13,14}$, the strong interaction does not play a role. The electromagnetic (EM) field strengths of relativistic ions scale with the proton number $(Z)$. For example, for a lead $(\mathrm{Pb})$ nucleus with $Z=82$ the field can be up to $10^{25} \mathrm{~V} \mathrm{~m}^{-1}$ (ref. 15), much larger than the Schwinger limit ${ }^{16}$ above which QED corrections become important. In the 1930s it was found that highly relativistic charged particles can be described by the equivalent photon approximation (EPA) ${ }^{17-19}$, which is schematically shown in Fig. 1b. The EM fields produced by the colliding $\mathrm{Pb}$ nuclei can be treated as a beam of quasi-real photons with a small virtuality of $Q^{2}<1 / R^{2}$, where $R$ is the radius of the charge distribution and so $Q^{2}<10^{-3} \mathrm{GeV}^{2}$. Then, the cross-section for the reaction $\mathrm{Pb}+\mathrm{Pb}(\gamma \gamma) \rightarrow \mathrm{Pb}+\mathrm{Pb} \gamma \gamma$ can be calculated by convolving the respective photon flux with the elementary cross-section for the process $\gamma \gamma \rightarrow \gamma \gamma$. Since the photon flux associated with each nucleus scales as $Z^{2}$, the cross-section is extremely enhanced as compared with proton-proton $(p p)$ collisions.

In this article, a measurement of LbyL scattering in $\mathrm{Pb}+\mathrm{Pb}$ collisions at the Large Hadron Collider (LHC) is reported, following the approach recently proposed in ref. 20 . The final-state signature of interest is the exclusive production of two photons, $\mathrm{Pb}+\mathrm{Pb}$ $(\gamma \gamma) \rightarrow \mathrm{Pb}^{(*)}+\mathrm{Pb}^{(*)} \gamma \gamma$, where a possible EM excitation of the outgoing ions ${ }^{21}$ is denoted by $\left({ }^{*}\right)$. Hence, the expected signature is two photons and no further activity in the central detector, since the $\mathrm{Pb}^{(*)}$ ions escape into the LHC beam pipe. Moreover, it is predicted that the background is relatively low in heavy-ion collisions and is dominated by exclusive dielectron $\left(\gamma \gamma \rightarrow e^{+} e^{-}\right)$production $^{20,22}$. The misidentification of electrons as photons can occur when the electron track is not reconstructed or the electron emits a hardbremsstrahlung photon. The fiducial cross-section of the process $\gamma \gamma \rightarrow \gamma \gamma$ in $\mathrm{Pb}+\mathrm{Pb}$ collisions is measured, using a data set recorded at a nucleon-nucleon centre-of-mass energy $\left(\sqrt{s_{\mathrm{NN}}}\right)$ of $5.02 \mathrm{TeV}$. This data set was recorded with the ATLAS detector at the LHC in 2015 and corresponds to an integrated luminosity of $480 \pm 30 \mu \mathrm{b}^{-1}$. In addition to the measured fiducial cross-section, the significance of the observed number of signal candidate events is given, assuming the background-only hypothesis.

\section{Experimental set-up}

ATLAS is a cylindrical particle detector composed of several subdetectors ${ }^{23}$. ATLAS uses a right-handed coordinate system with its origin at the nominal interaction point in the centre of the detector and the $z$ axis along the beam pipe. The $x$ axis points from the interaction point to the centre of the LHC ring, and the $y$ 
a

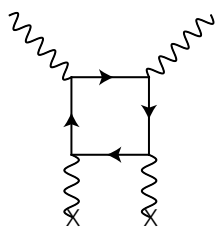

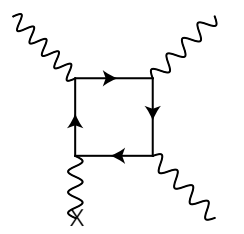

b

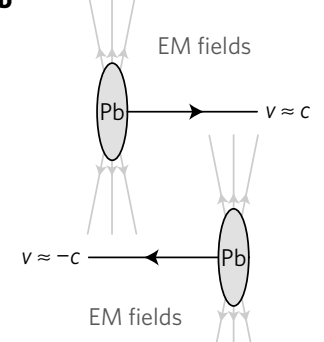

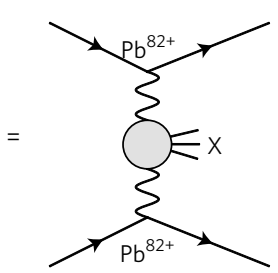

Figure 1 | Diagrams illustrating the QED LbyL interaction processes and the equivalent photon approximation. a, Diagrams for Delbrück scattering (left), photon splitting (middle) and elastic LbyL scattering (right). Each cross denotes external field legs, for example, an atomic Coulomb field or a strong background magnetic field. $\mathbf{b}$, Illustration of an ultra-peripheral collision of two lead ions. Electromagnetic interaction between the ions can be described as an exchange of photons that can couple to form a given final state $X$. The flux of photons is determined from the Fourier transform of the electromagnetic field of the ion, taking into account the nuclear electromagnetic form factors.

axis points upwards. Cylindrical coordinates $(r, \phi)$ are used in the transverse plane, with $\phi$ being the azimuthal angle around the $z$ axis. The pseudorapidity is defined in terms of the polar angle $\theta$ as $\eta=-\ln \tan (\theta / 2)$.

Angular distance is measured in units of $\Delta R \equiv \sqrt{(\Delta \eta)^{2}+(\Delta \phi)^{2}}$. The photon or electron transverse energy is $E_{\mathrm{T}}=E \sin (\theta)$, where $E$ is its energy. The inner tracking detector (ITD) consists of a silicon pixel system, a silicon microstrip detector and a straw-tube tracker immersed in a $2 \mathrm{~T}$ magnetic field provided by a superconducting solenoid. The ITD track reconstruction efficiency is estimated in ref. 24 for minimum-bias $p p$ events that, like UPC $\mathrm{Pb}+\mathrm{Pb}$ events, have a low average track multiplicity. For charged hadrons in the transverse momentum range $100<p_{\mathrm{T}}<200 \mathrm{MeV}$ the efficiency is about $50 \%$ and grows to $80 \%$ for $p_{\mathrm{T}}>200 \mathrm{MeV}$. Around the tracker there is a system of EM and hadronic calorimeters, which use liquid argon and lead, copper or tungsten absorbers for the EM and forward $(|\eta|>1.7)$ hadronic components of the detector, and scintillator-tile active material and steel absorbers for the central $(|\eta|<1.7)$ hadronic component. The muon spectrometer consists of separate trigger and high-precision tracking chambers measuring the trajectory of muons in a magnetic field generated by superconducting air-core toroids. The ATLAS minimum-bias trigger scintillators (MBTSs) consist of scintillator slabs positioned between the ITD and the endcap calorimeters with each side having an outer ring of four slabs segmented in azimuthal angle, covering $2.07<|\eta|<2.76$ and an inner ring of eight slabs, covering $2.76<|\eta|<3.86$. The ATLAS zero-degree calorimeters (ZDCs), located along the beam axis at $140 \mathrm{~m}$ from the interaction point on both sides, detect neutral particles (including neutrons emitted from the nucleus). The ATLAS trigger system ${ }^{25}$ consists of a Level-1 trigger implemented using a combination of dedicated electronics and programmable logic, and a software-based high-level trigger.

\section{Monte Carlo simulation and theoretical predictions}

Several Monte Carlo (MC) samples are produced to estimate background contributions and corrections to the fiducial measurement. The detector response is modelled using a simulation based on GEANT4 (refs 26,27). The data and MC simulated events are passed through the same reconstruction and analysis procedures.

LbyL signal events are generated taking into account box diagrams with charged leptons and quarks in the loops, as detailed in ref. 28. The contributions from $W$-boson loops are omitted in the calculations since they are mostly important for diphoton masses $m_{\gamma \gamma}>2 m_{W}$ (ref. 29). The calculations are then convolved with the $\mathrm{Pb}+\mathrm{Pb}$ EPA spectrum from the STARlight 1.1 MC generator ${ }^{30}$. Next, various diphoton kinematic distributions are cross-checked with predictions from ref. 20 and good agreement is found. The theoretical uncertainty on the cross-section is mainly due to limited knowledge of the nuclear electromagnetic form factors and the related initial photon fluxes. This is studied in ref. 20 and the relevant uncertainty is conservatively estimated to be $20 \%$. Higher-order corrections (not included in the calculations) are also part of the theoretical uncertainty and are of the order of a few per cent for diphoton invariant masses below $100 \mathrm{GeV}$ (refs 31,32).

The sources of background considered in this analysis are: $\gamma \gamma \rightarrow e^{+} e^{-}$, central exclusive production (CEP) of photon pairs, exclusive production of quark-antiquark pairs $(\gamma \gamma \rightarrow q \bar{q})$ and other backgrounds that could mimic the diphoton event signatures. The $\gamma \gamma \rightarrow e^{+} e^{-}$background is modelled with STARlight 1.1 (ref. 30), in which the cross-section is computed by combining the $\mathrm{Pb}+\mathrm{Pb}$ EPA with the leading-order formula for $\gamma \gamma \rightarrow e^{+} e^{-}$. This process has been recently measured by the ALICE Collaboration, and a good agreement with STARlight is found ${ }^{33}$. The exclusive diphoton final state can be also produced via the strong interaction through a quark loop in the exchange of two gluons in a colour-singlet state (see Supplementary Fig. 2). This CEP process, $g g \rightarrow \gamma \gamma$, is modelled using SupERCHIC 2.03 (ref. 34), in which the $p p$ crosssection has been scaled by $A^{2} R_{\mathrm{g}}^{4}$ as suggested in ref. 20 , where $A=208$ and $R_{\mathrm{g}} \approx 0.7$ is a gluon shadowing correction $^{35}$. This process has a large theoretical uncertainty, of $\mathcal{O}(100 \%)$, mostly related to incomplete knowledge of gluon densities ${ }^{36}$. The $\gamma \gamma \rightarrow q \bar{q}$ contribution is estimated using Herwig ++2.7 .1 (ref. 37) where the EPA formalism in $p p$ collisions is implemented. The $\gamma \gamma \rightarrow q \bar{q}$ sample is then normalized to the corresponding cross-section in $\mathrm{Pb}+\mathrm{Pb}$ collisions ${ }^{30}$.

\section{Event selection}

Candidate diphoton events were recorded in the $\mathrm{Pb}+\mathrm{Pb}$ run in 2015 using a dedicated trigger for events with moderate activity in the calorimeter but little additional activity in the entire detector. At Level-1 the total $E_{\mathrm{T}}$ registered in the calorimeter after noise suppression was required to be between 5 and $200 \mathrm{GeV}$. Then at the high-level trigger, events were rejected if more than one hit was found in the inner ring of the MBTS (MBTS veto) or if more than ten hits were found in the pixel detector.

The efficiency of the Level-1 trigger is estimated with $\gamma \gamma \rightarrow e^{+} e^{-}$ events passing an independent supporting trigger. This trigger is designed to select events with mutual dissociation of $\mathrm{Pb}$ nuclei and small activity in the ITD. It is based on a coincidence of signals in both $\mathrm{ZDC}$ sides and a requirement on the total $E_{\mathrm{T}}$ in the calorimeter below $50 \mathrm{GeV}$. Event candidates are required to have only two reconstructed tracks and two EM energy clusters. Furthermore, to reduce possible backgrounds, each pair of clusters $(\mathrm{cl} 1, \mathrm{cl} 2)$ is required to have a small acoplanarity $\left(1-\Delta \phi_{\mathrm{cl} 1, \mathrm{cl} 2} / \pi<0.2\right)$. The extracted Level-1 trigger efficiency is provided as a function of the 

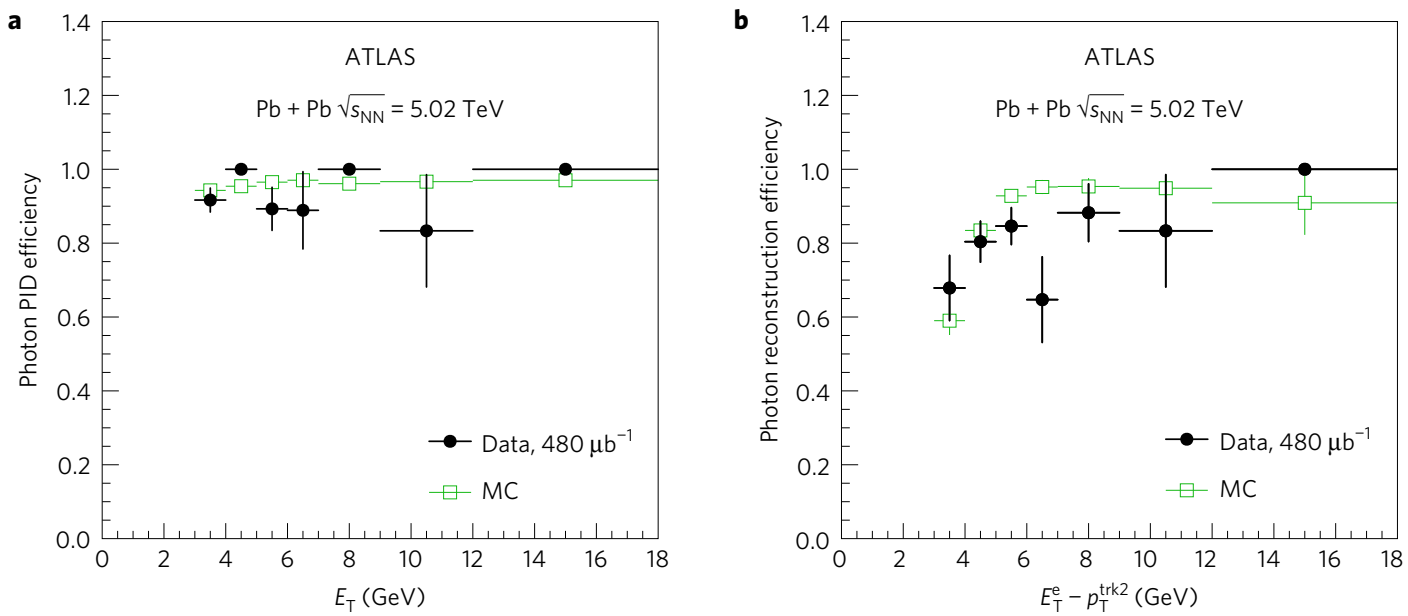

Figure 2 | Photon identification and reconstruction efficiencies. a, Photon PID efficiency as a function of photon $E_{\mathrm{T}}$ extracted from FSR event candidates. b. Photon reconstruction efficiency as a function of photon $E_{T}$ (approximated with $E_{T}^{e}-p_{\top}^{\text {trk2 }}$ ) extracted from $\gamma \gamma \rightarrow e^{+} e^{-}$events with a hard-bremsstrahlung photon. Data (filled markers) are compared with MC simulations (open markers). The statistical uncertainties arising from the finite size of the data and simulation samples are indicated by vertical bars.

sum of cluster transverse energies $\left(E_{\mathrm{T}}^{\mathrm{cl} 1}+E_{\mathrm{T}}^{\mathrm{c} 2}\right)$. The efficiency grows from about $70 \%$ at $\left(E_{\mathrm{T}}^{\mathrm{cl} 1}+E_{\mathrm{T}}^{\mathrm{c} 2}\right)=6 \mathrm{GeV}$ to $100 \%$ at $\left(E_{\mathrm{T}}^{\mathrm{cl} 1}+E_{\mathrm{T}}^{\mathrm{c} 2}\right)>$ $9 \mathrm{GeV}$. The efficiency is parameterized using an error function fit, which is then used to reweight the simulation. Due to the extremely low noise, very high hit reconstruction efficiency and low conversion probability of signal photons in the pixel detector (around 10\%), the uncertainty due to the requirement for minimal activity in the ITD is negligible. The MBTS veto efficiency was studied using $\gamma \gamma \rightarrow \ell^{+} \ell^{-}$events $(\ell=e, \mu)$ passing a supporting trigger and it is estimated to be $(98 \pm 2) \%$.

Photons are reconstructed from EM clusters in the calorimeter and tracking information provided by the ITD, which allows the identification of photon conversions. Selection requirements are applied to remove EM clusters with a large amount of energy from poorly functioning calorimeter cells, and a timing requirement is made to reject out-of-time candidates. An energy calibration specifically optimized for photons ${ }^{38}$ is applied to the candidates to account for upstream energy loss and both lateral and longitudinal shower leakage. A dedicated correction $^{39}$ is applied for photons in MC samples to correct for potential mismodelling of quantities that describe the properties ('shapes') of the associated EM showers.

The photon particle identification (PID) in this analysis is based on three shower-shape variables: the lateral width of the shower in the middle layer of the EM calorimeter, the ratio of the energy difference associated with the largest and second largest energy deposits to the sum of these energies in the first layer, and the fraction of energy reconstructed in the first layer relative to the total energy of the cluster. Only photons with $E_{\mathrm{T}}>3 \mathrm{GeV}$ and $|\eta|<2.37$, excluding the calorimeter transition region $1.37<|\eta|<1.52$, are considered. The pseudorapidity requirement ensures that the photon candidates pass through regions of the EM calorimeter where the first layer is segmented into narrow strips, allowing for good separation between genuine prompt photons and photons coming from the decay of neutral hadrons. A constant photon PID efficiency of $95 \%$ as a function of $\eta$ with respect to reconstructed photon candidates is maintained. This is optimized using multivariate analysis techniques ${ }^{40}$, such that EM energy clusters induced by cosmic-ray muons are rejected with $95 \%$ efficiency.

Preselected events are required to have exactly two photons satisfying the above selection criteria, with a diphoton invariant mass greater than $6 \mathrm{GeV}$. To reduce the dielectron background, a veto on the presence of any charged-particle tracks (with $p_{\mathrm{T}}>100 \mathrm{MeV},|\eta|<2.5$ and at least one hit in the pixel detector) is imposed. This requirement further reduces the fake-photon background from the dielectron final state by a factor of 25 , according to simulation. It has almost no impact on $\gamma \gamma \rightarrow \gamma \gamma$ signal events, since the probability of photon conversion in the pixel detector is relatively small and converted photons are suppressed at low $E_{\mathrm{T}}(3-6 \mathrm{GeV})$ by the photon selection requirements. According to $\mathrm{MC}$ studies, the photon selection requirements remove about $10 \%$ of low- $E_{\mathrm{T}}$ photons. To reduce other fake-photon backgrounds (for example, cosmic-ray muons), the transverse momentum of the diphoton system $\left(p_{\mathrm{T}}^{\gamma \gamma}\right)$ is required to be below $2 \mathrm{GeV}$. To reduce background from CEP $g g \rightarrow \gamma \gamma$ reactions, an additional requirement on diphoton acoplanarity, Aco $=1-\Delta \phi_{\gamma \gamma} / \pi<0.01$, is imposed. This requirement is optimized to retain a high signal efficiency and reduce the CEP background significantly, since the transverse momentum transferred by the photon exchange is usually much smaller than that due to the colour-singlet-state gluons ${ }^{41}$.

\section{Performance and validation of photon reconstruction}

Since the analysis requires the presence of low-energy photons, which are not typically used in ATLAS analyses, detailed studies of photon reconstruction and calibration are performed.

High- $p_{\mathrm{T}} \gamma \gamma \rightarrow \ell^{+} \ell^{-}$production with a final-state radiation (FSR) photon is used for the measurement of the photon PID efficiency. Events with a photon and two tracks corresponding to oppositely charged particles with $p_{\mathrm{T}}>1 \mathrm{GeV}$ are required to pass the same trigger as in the diphoton selection or the supporting trigger. The $\Delta R$ between a photon candidate and a track is required to be greater than 0.2 to avoid leakage of the electron clusters from the $\gamma \gamma \rightarrow e^{+} e^{-}$process to the photon cluster. The FSR event candidates are identified using a $p_{\mathrm{T}}^{\mathrm{tt} \gamma}<1 \mathrm{GeV}$ requirement, where $p_{\mathrm{T}}^{\mathrm{t} \gamma}$ is the transverse momentum of the three-body system consisting of two charged-particle tracks and a photon. The FSR photons are then used to extract the photon PID efficiency, which is defined as the probability for a reconstructed photon to satisfy the identification criteria. Figure 2a shows the photon PID efficiencies in data and simulation as a function of reconstructed photon $E_{\mathrm{T}}$. Within their statistical precision the two results agree.

The photon reconstruction efficiency is extracted from data using $\gamma \gamma \rightarrow e^{+} e^{-}$events where one of the electrons emits a hard-bremsstrahlung photon due to interaction with the material of the detector. Events with exactly one identified electron, two reconstructed charged-particle tracks and exactly one photon are studied. The electron $E_{\mathrm{T}}$ is required to be above $5 \mathrm{GeV}$ and the $p_{\mathrm{T}}$ 

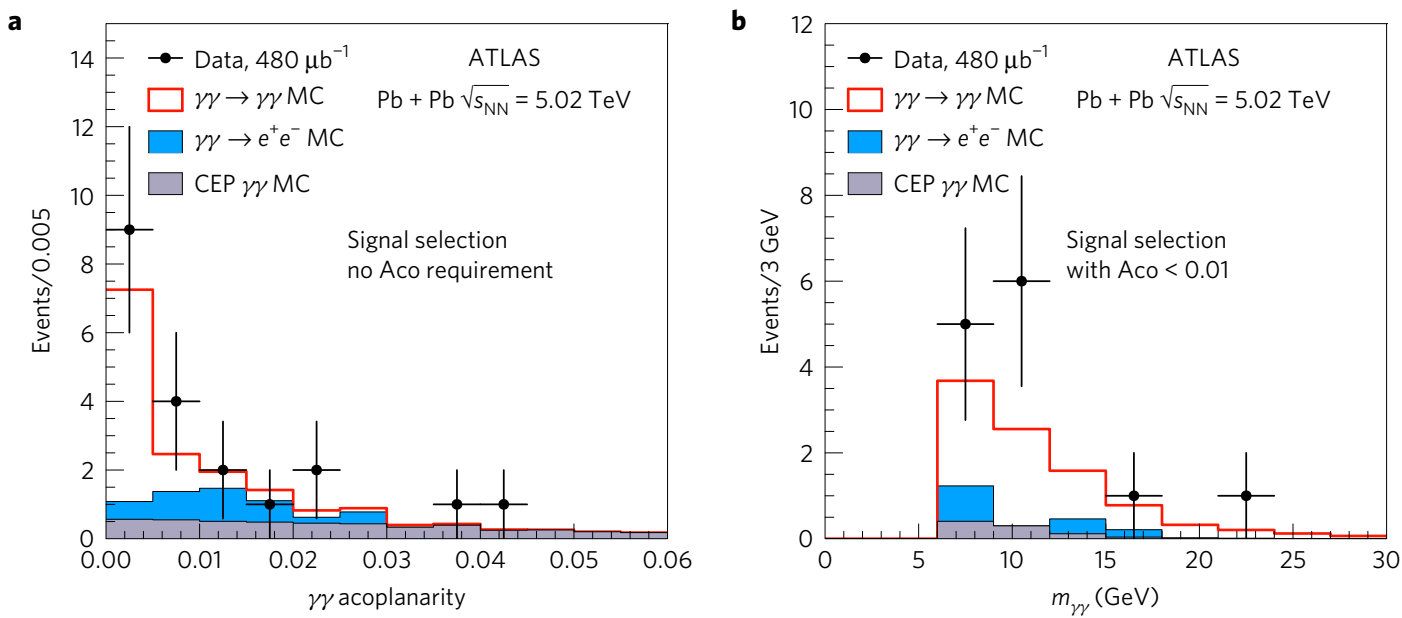

Figure 3 | Kinematic distributions for $\gamma \gamma \rightarrow \gamma \gamma$ event candidates. a, Diphoton acoplanarity before applying the Aco $<0.01$ requirement. b, Diphoton invariant mass after applying the Aco $<0.01$ requirement. Data (points) are compared to $\mathrm{MC}$ predictions (histograms). The statistical uncertainties on the data are shown as vertical bars.

of the track that is unmatched with the electron (trk2) is required to be below $2 \mathrm{GeV}$. The additional hard-bremsstrahlung photon is expected to have $E_{\mathrm{T}}^{\gamma} \approx\left(E_{\mathrm{T}}^{e}-p_{\mathrm{T}}^{\mathrm{trk} 2}\right)$. The $p_{\mathrm{T}}^{\mathrm{trk} 2}<2 \mathrm{GeV}$ requirement ensures a sufficient $\Delta R$ separation between the expected photon and the second electron, extrapolated to the first layer of the EM calorimeter. The data sample contains $247 \gamma \gamma \rightarrow e^{+} e^{-}$events that are used to extract the photon reconstruction efficiency, which is presented in Fig. 2b. Good agreement between data and $\gamma \gamma \rightarrow e^{+} e^{-}$ MC simulation is observed and the photon reconstruction efficiency is measured with a $5-10 \%$ relative uncertainty at low $E_{\mathrm{T}}(3-6 \mathrm{GeV})$.

In addition, a cross-check is performed on $Z \rightarrow \mu^{+} \mu^{-} \gamma$ events identified in $p p$ collision data from 2015 corresponding to an integrated luminosity of $1.6 \mathrm{fb}^{-1}$. The results support (in a similar way to ref. 42) the choice to use the three shower-shape variables in this photon PID selection in an independent sample of low- $E_{\mathrm{T}}$ photons.

The photon cluster energy resolution is extracted from data using $\gamma \gamma \rightarrow e^{+} e^{-}$events. The electrons from the $\gamma \gamma \rightarrow e^{+} e^{-}$ reaction (see Supplementary Information) are well balanced in their transverse momenta, with very small standard deviation, $\sigma_{p_{\mathrm{T}}^{e+}-p_{\mathrm{T}}^{e-}}<$ $30 \mathrm{MeV}$, much smaller than the expected EM calorimeter energy resolution. Therefore, by measuring $\left(E_{\mathrm{T}}^{\mathrm{cl} 1}-E_{\mathrm{T}}^{\mathrm{c} 2}\right)$ distributions in $\gamma \gamma \rightarrow e^{+} e^{-}$events, one can extract the cluster energy resolution, $\sigma_{E_{\mathrm{T}}^{\mathrm{cl}}}$. For electrons with $E_{\mathrm{T}}<10 \mathrm{GeV}$, the $\sigma_{E_{\mathrm{T}}^{\mathrm{cl}}} / E_{\mathrm{T}}^{\mathrm{cl}}$ is observed to be approximately $8 \%$ both in data and simulation. An uncertainty of $\delta \sigma_{E_{\mathrm{T}}^{\gamma}} / \sigma_{E_{\mathrm{T}}^{\gamma}}=15 \%$ is assigned to the simulated photon energy resolution and takes into account differences between $\sigma_{E_{T}^{c l}}$ in data and $\sigma_{E_{T}^{\gamma}}$ in simulation.

Similarly, the EM cluster energy scale can be studied using the $\left(E_{\mathrm{T}}^{\mathrm{cl} 1}+E_{\mathrm{T}}^{\mathrm{c} 2}\right)$ distribution. It is observed that the simulation provides a good description of this distribution, within the relative uncertainty of $5 \%$ that is assigned to the EM cluster energy-scale modelling.

\section{Background estimation}

Due to its relatively high rate, the exclusive production of electron pairs $\left(\gamma \gamma \rightarrow e^{+} e^{-}\right)$can be a source of fake diphoton events. The contribution from the dielectron background is estimated using $\gamma \gamma \rightarrow e^{+} e^{-}$MC simulation (which gives 1.3 events) and is verified using the following data-driven technique. Two control regions are defined that are expected to be dominated by $\gamma \gamma \rightarrow e^{+} e^{-}$ backgrounds. The first control region is defined by requiring events with exactly one reconstructed charged-particle track and two identified photons that satisfy the same preselection criteria as for the signal definition. The second control region is defined similarly to the first one, except exactly two tracks are required $\left(N_{\text {trk }}=2\right)$. Good agreement is observed between data and MC simulation in both control regions, but the precision is limited by the number of events in data. A conservative uncertainty of $25 \%$ is therefore assigned to the $\gamma \gamma \rightarrow e^{+} e^{-}$background estimation, which reflects the statistical uncertainty of data in the $N_{\text {trk }}=1$ control region. The contribution from a related QED process, $\gamma \gamma \rightarrow e^{+} e^{-} \gamma \gamma$, is evaluated using the MadGraph5_aMC@NLO MC generator ${ }^{43}$ and is found to be negligible.

The Aco $<0.01$ requirement significantly reduces the CEP $g g \rightarrow \gamma \gamma$ background. However, the MC prediction for this process has a large theoretical uncertainty; hence, an additional data-driven normalization is performed in the region Aco $>b$, where $b$ is a value greater than 0.01 which can be varied. Three values of $b(0.01,0.02$, 0.03 ) are used, where the central value $b=0.02$ is chosen to derive the nominal background prediction and the values $b=0.01$ and $b=0.03$ to define the systematic uncertainty. The normalization is performed using the condition: $f_{g g \rightarrow \gamma \gamma \gamma}^{\text {norm } b}=\left(N_{\text {data }}(\right.$ Aco $>b)-N_{\text {sig }}$ $($ Aco $>b)-N_{\gamma \gamma \rightarrow e^{+} e^{-}}($Aco $\left.>b)\right) / N_{g g \rightarrow \gamma \gamma}($ Aco $>b)$, for each value of $b$, where $N_{\text {data }}$ is the number of observed events, $N_{\text {sig }}$ is the expected number of signal events, $N_{\gamma \gamma \rightarrow e^{+} e^{-}}$is the expected background from $\gamma \gamma \rightarrow e^{+} e^{-}$events and $N_{g g \rightarrow \gamma \gamma}$ is the MC estimate of the expected background from CEP $g g \rightarrow \gamma \gamma$ events. The normalization factor is found to be $f_{g g \rightarrow \gamma \gamma}^{\text {norm }}=0.5 \pm 0.3$ and the background due to CEP $g g \rightarrow \gamma \gamma$ is estimated to be $f_{g g \rightarrow \gamma \gamma}^{\text {norm }} \times N_{g g \rightarrow \gamma \gamma}($ Aco $<0.01)=0.9 \pm$ 0.5 events. To verify the CEP $g g \rightarrow \gamma \gamma$ background estimation method, energy deposits in the ZDC are studied for events before the Aco selection. It is expected that the outgoing ions in CEP events predominantly dissociate, which results in the emission of neutrons detectable in the $\mathrm{ZDC}^{20}$. Good agreement between the normalized CEP $g g \rightarrow \gamma \gamma$ MC expectation and the observed events with a ZDC signal corresponding to at least 1 neutron is observed in the full Aco range (see Supplementary Information for details).

Low- $p_{\mathrm{T}}$ dijet events can produce multiple $\pi^{0}$ mesons, which could potentially mimic diphoton events. The event selection requirements are efficient in rejecting such events, and based on studies performed with a supporting trigger, the background from hadronic processes is estimated to be $0.3 \pm 0.3$ events. MC studies show that the background from $\gamma \gamma \rightarrow q \bar{q}$ processes is negligible.

Exclusive neutral two-meson production can be a potential source of background for LbyL events, mainly due to their back-toback topology being similar to that of the CEP $g g \rightarrow \gamma \gamma$ process. The cross-section for this process is calculated to be below $10 \%$ of the CEP $g g \rightarrow \gamma \gamma$ cross-section $^{44,45}$ and it is therefore considered to 
Table 1 | The number of events accepted by the sequential selection requirements for data, compared with the number of background and signal events expected from the simulation.

\begin{tabular}{llllllll} 
Selection & $\boldsymbol{\gamma} \boldsymbol{\gamma} \rightarrow \mathbf{e}^{+} \mathbf{e}^{-}$ & CEP $\boldsymbol{g g} \rightarrow \boldsymbol{\gamma} \boldsymbol{\gamma}$ & Hadronic fakes & Other fakes & Total background & Signal & Data \\
\hline Preselection & 74 & 4.7 & 6 & 19 & 104 & 9.1 \\
$N_{\text {trk }}=0$ & 4.0 & 4.5 & 6 & 19 & 33 & 105 \\
$p_{\mathrm{T}}^{\gamma \gamma}<2 \mathrm{GeV}$ & 3.5 & 4.4 & 3 & 1.3 & 12.2 & 39 \\
Aco $<0.01$ & 1.3 & 0.9 & 0.3 & 0.1 & 2.6 & 8.5 \\
Uncertainty & 0.3 & 0.5 & 0.3 & 0.1 & 0.7 & 7.3 \\
\hline
\end{tabular}

The signal simulation is based on calculations from ref. 28. In addition, the uncertainties on the expected number of events passing all selection requirements are given

give a negligible contribution to the signal region. The contribution from bottomonia production (for example, $\gamma \gamma \rightarrow \eta_{\mathrm{b}} \rightarrow \gamma \gamma$ or $\left.\gamma \mathrm{Pb} \rightarrow \Upsilon \rightarrow \gamma \eta_{\mathrm{b}} \rightarrow 3 \gamma\right)$ is calculated using parameters from refs 46 , 47 and is found to be negligible.

The contribution from other fake diphoton events (for example those induced by cosmic-ray muons) is estimated using photons that fail to satisfy the longitudinal shower-shape requirement. The total background due to other fake photons is found to be $0.1 \pm 0.1$ events. As a further cross-check, additional activity in the muon spectrometer is studied. It is observed that out of 18 events satisfying the inverted $p_{\mathrm{T}}^{\gamma \gamma}$ requirement, 13 have at least one additional reconstructed muon. In the region $p_{\mathrm{T}}^{\gamma \gamma}<2 \mathrm{GeV}$, no events with muon activity are found, which is compatible with the abovementioned estimate of $0.1 \pm 0.1$.

The contribution from UPC events where both nuclei emit a bremsstrahlung photon is estimated using calculations from ref. 13 and is found to be negligible for photons with $|\eta|<2.4$ and $E_{\mathrm{T}}>3 \mathrm{GeV}$.

\section{Results}

Photon kinematic distributions for events satisfying the selection criteria are shown in Fig. 3. The shape of the diphoton acoplanarity distribution for $\gamma \gamma \rightarrow e^{+} e^{-}$events in Fig. 3a reflects the trajectories of the electron and positron in the detector magnetic field, before they emit hard photons in their collisions with the ITD material. In total, 13 events are observed in data whereas 7.3 signal events and 2.6 background events are expected. In general, good agreement between data and MC simulation is observed. The effect of sequential selection requirements on the number of events selected is shown in Table 1, for each of the data, signal and background samples.

To quantify an excess of events over the background expectation, a test statistic based on the profile likelihood ratio ${ }^{48}$ is used. The $p$ value for the background-only hypothesis, defined as the probability for the background to fluctuate and give an excess of events as large or larger than that observed in the data, is found to be $5 \times 10^{-6}$. The $p$ value can be expressed in terms of Gaussian tail probabilities, which, given in units of standard deviation $(\sigma)$, corresponds to a significance of $4.4 \sigma$. The expected $p$ value and significance (obtained before the fit of the signal-plus-background hypothesis to the data and using standard model predictions from ref. 28 ) are $8 \times 10^{-5}$ and $3.8 \sigma$, respectively.

The cross-section for the $\mathrm{Pb}+\mathrm{Pb}(\gamma \gamma) \rightarrow \mathrm{Pb}^{(*)}+\mathrm{Pb}^{(*)} \gamma \gamma$ process is measured in a fiducial phase space defined by the photon transverse energy $E_{\mathrm{T}}>3 \mathrm{GeV}$, photon absolute pseudorapidity $|\eta|<2.4$, diphoton invariant mass greater than $6 \mathrm{GeV}$, diphoton transverse momentum lower than $2 \mathrm{GeV}$ and diphoton acoplanarity below 0.01 . Experimentally, the fiducial cross-section is given by

$$
\sigma_{\text {fid }}=\frac{N_{\text {data }}-N_{\mathrm{bkg}}}{C \times \int L \mathrm{~d} t}
$$

where $N_{\text {data }}$ is the number of selected events in data, $N_{\text {bkg }}$ is the expected number of background events and $\int L \mathrm{~d} t$ is the integrated
Table 2 | Summary of systematic uncertainties.

\begin{tabular}{ll} 
Source of uncertainty & $\begin{array}{l}\text { Relative } \\
\text { uncertainty }\end{array}$ \\
\hline Trigger & $5 \%$ \\
Photon reco. efficiency & $12 \%$ \\
Photon PID efficiency & $16 \%$ \\
Photon energy scale & $7 \%$ \\
Photon energy resolution & $11 \%$ \\
Total & $24 \%$ \\
\hline The table shows the relative systematic uncertainty on detector correction factor C broken into \\
its individual contributions. The total is obtained by adding them in quadrature.
\end{tabular}

luminosity. The factor $C$ is used to correct for the net effect of the trigger efficiency, the diphoton reconstruction and PID efficiencies, as well as the impact of photon energy and angular resolution. It is defined as the ratio of the number of generated signal events satisfying the selection criteria after particle reconstruction and detector simulation to the number of generated events satisfying the fiducial criteria before reconstruction. The value of $C$ and its total uncertainty is determined to be $0.31 \pm 0.07$. The dominant systematic uncertainties come from the uncertainties on the photon reconstruction and identification efficiencies. Other minor sources of uncertainty are the photon energy scale and resolution uncertainties and trigger efficiency uncertainty. To check for a potential model dependence, calculations from ref. 28 are compared with predictions from ref. 20, and a negligible impact on the $C$-factor uncertainty is found. Table 2 lists the separate contributions to the systematic uncertainty. The uncertainty on the integrated luminosity is $6 \%$. It is derived following a methodology similar to that detailed in refs 49,50 , from a calibration of the luminosity scale using $x-y$ beam-separation scans performed in December 2015.

The measured fiducial cross-section is $\sigma_{\text {fid }}=70 \pm 24$ (stat.) \pm 17 (syst.) nb, which is in agreement with the predicted values of $45 \pm 9 \mathrm{nb}$ (ref. 20) and $49 \pm 10 \mathrm{nb}$ (ref. 28) within uncertainties.

\section{Conclusion}

In summary, this article presents evidence for the scattering of LbyL in quasi-real photon interactions from $480 \mu \mathrm{b}^{-1}$ of ultra-peripheral $\mathrm{Pb}+\mathrm{Pb}$ collisions at $\sqrt{s_{\mathrm{NN}}}=5.02 \mathrm{TeV}$ by the ATLAS experiment at the LHC. The statistical significance against the background-only hypothesis is found to be 4.4 standard deviations. After background subtraction and analysis corrections, the fiducial cross-section for the $\mathrm{Pb}+\mathrm{Pb}(\gamma \gamma) \rightarrow \mathrm{Pb}^{(*)}+\mathrm{Pb}^{(*)} \gamma \gamma$ process was measured and is compatible with standard model predictions.

The analysis is mostly limited by the amount of data available and the lower limit on transverse energy for reconstructed photons $\left(E_{\mathrm{T}}=3 \mathrm{GeV}\right)$, below which more signal is expected. Advancements on these two points would also allow for reconstruction of low-mass mesons decaying into two photons, which in turn could be used to improve detector calibration. The heavy-ion data yield is expected to double at the end of 2018 (and again increase tenfold after 
LHC Run 4, scheduled to start in 2026), which would significantly reduce the statistical uncertainty. Future upgrades of ATLAS, such as extended tracking acceptance from $|\eta|<2.5$ to $|\eta|<4.0$, will further improve this.

Data availability. The experimental data that support the findings of this study are available in HEPData with the identifier http://dx.doi.org/10.17182/hepdata.77761.

\section{Received 9 February 2017; accepted 15 June 2017; published online 14 August 2017}

\section{References}

1. Heisenberg, W. \& Euler, H. Folgerungen aus der Diracschen Theorie des Positrons. Z. Phys. 98, 714-732 (1936).

2. King, B. \& Heinzl, T. Measuring vacuum polarisation with high power lasers. High Power Laser Sci. Eng. 4, e5 (2016).

3. Hanneke, D., Fogwell, S. \& Gabrielse, G. New measurement of the electron magnetic moment and the fine structure constant. Phys. Rev. Lett. 100, 120801 (2008).

4. Bennett, G.W. et al. (Muon $g-2$ Collaboration) Final report of the muon E821 anomalous magnetic moment measurement at BNL. Phys. Rev. D 73, 072003 (2006).

5. Laporta, S. \& Remiddi, E. The analytical value of the electron light-light graphs contribution to the muon $(g-2)$ in QED. Phys. Lett. B 301, 440-446 (1993).

6. Wilson, R. R. Scattering of $1.33 \mathrm{MeV}$ gamma-rays by an electric field. Phys. Rev. 90, 720-721 (1953)

7. Jarlskog, G. et al. Measurement of Delbrück scattering and observation of photon splitting at high energies. Phys. Rev. D 8, 3813-3823 (1973).

8. Schumacher, M. et al. Delbrück scattering of $2.75-\mathrm{MeV}$ photons by lead. Phys. Lett. B 59, 134-136 (1975).

9. Akhmadaliev, S. Z. et al. Delbrück scattering at energies of $140-450 \mathrm{MeV}$. Phys. Rev. C 58, 2844-2850 (1998).

10. Akhmadaliev, S. Z. et al. Experimental investigation of high-energy photon splitting in atomic fields. Phys. Rev. Lett. 89, 061802 (2002).

11. Burke, D. L. et al. Positron production in multi-photon light by light scattering. Phys. Rev. Lett. 79, 1626-1629 (1997).

12. Bula, C. et al. Observation of nonlinear effects in Compton scattering. Phys. Rev. Lett. 76, 3116-3119 (1996).

13. Bertulani, C. A. \& Baur, G. Electromagnetic processes in relativistic heavy ion collisions. Phys. Rep. 163, 299-408 (1988).

14. Natale, A. A., Roldao, C. G. \& Carneiro, J. P. V. Two photon final states in peripheral heavy ion collisions. Phys. Rev. C 65, 014902 (2002).

15. Șengül, M. Y. et al. Electromagnetic heavy-lepton pair production in relativistic heavy-ion collisions. Eur. Phys. J. C 76, 428 (2016).

16. Schwinger, J. S. On gauge invariance and vacuum polarization. Phys. Rev. 82, 664-679 (1951).

17. Fermi, E. On the theory of collisions between atoms and electrically charged particles. Nuovo Cimento 2, 143-158 (1925).

18. Weizsäcker, C. F. Radiation emitted in collisions of very fast electrons. Z. Phys. 88, 612-625 (1934)

19. Williams, E. J. Nature of the high energy particles of penetrating radiation and status of ionization and radiation formulae. Phys. Rev. 45, 729-730 (1934).

20. d'Enterria, D. \& Silveira, G. G. Observing light-by-light scattering at the large hadron collider. Phys. Rev. Lett. 111, 080405 (2013); erratum 116, 129901 (2016).

21. Abelev, B. et al. (ALICE Collaboration) Measurement of the cross section for electromagnetic dissociation with neutron emission in $\mathrm{Pb}-\mathrm{Pb}$ collisions at $\sqrt{s_{\mathrm{NN}}}=2.76 \mathrm{TeV}$. Phys. Rev. Lett. 109, 252302 (2012).

22. Knapen, S. et al. Searching for axionlike particles with ultraperipheral heavy-ion collisions. Phys. Rev. Lett. 118, 171801 (2017)

23. ATLAS Collaboration. The ATLAS experiment at the CERN large hadron collider. JINST 3, S08003 (2008).

24. ATLAS Collaboration. Charged-particle distributions at low transverse momentum in $\sqrt{s}=13 \mathrm{TeV}$ pp interactions measured with the ATLAS detector at the LHC. Eur. Phys. J. C 76, 502 (2016).

25. ATLAS Collaboration. Performance of the ATLAS trigger system in 2010 Eur. Phys. J. C 72, 1849 (2012).

26. Agostinelli, S. et al. GEANT4: a Simulation toolkit. Nucl. Instrum. Meth. A 506, 250-303 (2003).

27. ATLAS Collaboration. The ATLAS simulation infrastructure. Eur. Phys. J. C 70, 823-874 (2010)

28. Kłusek-Gawenda, M., Lebiedowicz, P. \& Szczurek, A. Light-by-light scattering in ultraperipheral $\mathrm{Pb}-\mathrm{Pb}$ collisions at energies available at the CERN Large Hadron Collider. Phys. Rev. C 93, 044907 (2016).
29. Fichet, S. et al. Light-by-light scattering with intact protons at the LHC: from standard model to new physics. J. High Energy Phys. 2, 165 (2015).

30. Klein, S. R. et al. STARlight: a Monte Carlo simulation program for ultra-peripheral collisions of relativistic ions. Comput. Phys. Commun. 212, 258-268 (2017)

31. Bern, Z. et al. QCD and QED corrections to light by light scattering. J. High Energy Phys. 11, 031 (2001).

32. Kłusek-Gawenda, M., Schäfer, W. \& Szczurek, A. Two-gluon exchange contribution to elastic $\gamma \gamma \rightarrow \gamma \gamma$ scattering and production of two-photons in ultraperipheral ultrarelativistic heavy ion and proton-proton collisions. Phys. Lett. B 761, 399-407 (2016).

33. Abbas, E. et al. (ALICE Collaboration) Charmonium and $\mathrm{e}^{+} \mathrm{e}^{-}$pair photoproduction at mid-rapidity in ultra-peripheral $\mathrm{Pb}-\mathrm{Pb}$ collisions at $\sqrt{s_{\mathrm{NN}}}=2.76$ TeV. Eur. Phys. J. C 73, 2617 (2013).

34. Harland-Lang, L. A., Khoze, V. A. \& Ryskin, M. G. Exclusive physics at the LHC with SuperChic 2. Eur. Phys. J. C 76, 9 (2016)

35. Eskola, K. J., Paukkunen, H. \& Salgado, C. A. EPS09: a new generation of NLO and LO nuclear parton distribution functions. J. High Energy Phys. 4, 065 (2009).

36. Aaltonen, T. et al. (CDF Collaboration) Observation of exclusive gamma gamma production in $p \bar{p}$ collisions at $\sqrt{s}=1.96 \mathrm{TeV}$. Phys. Rev. Lett. 108, 081801 (2012)

37. Bähr, M. et al. Herwig++ physics and manual. Eur. Phys. J. C 58, 639-707 (2008)

38. ATLAS Collaboration. Electron and photon energy calibration with the ATLAS detector using LHC Run 1 data. Eur. Phys. J. C 74, 3071 (2014).

39. ATLAS Collaboration. Measurement of the photon identification efficiencies with the ATLAS detector using LHC Run-1 data. Eur. Phys. J. C 76, 666 (2016).

40. Höcker, A. et al. TMVA - toolkit for multivariate data analysis. PoS ACAT Preprint at http://arXiv.org/abs/physics/0703039 (2007)

41. Baur, G. et al. Coherent gamma gamma and gamma-A interactions in very peripheral collisions at relativistic ion colliders. Phys. Rep. 364, 359-450 (2002).

42. ATLAS Collaboration. Reconstruction of Collinear Final-State-Radiation Photons in $Z$ Decays to Muons in $\sqrt{s}=7$ TeV Proton-Proton Collisions (ATLAS-CONF-2012-143, 2012); https://cds.cern.ch/record/1491697

43. Alwall, J. et al. The automated computation of tree-level and next-to-leading order differential cross sections, and their matching to parton shower simulations. J. High Energy Phys. 7, 79 (2014).

44. Harland-Lang, L. A. et al. Central exclusive meson pair production in the perturbative regime at hadron colliders. Eur. Phys. J. C 71, 1714 (2011).

45. Harland-Lang, L. A. et al. Central exclusive production as a probe of the gluonic component of the eta' and eta mesons. Eur. Phys. J. C 73, 2429 (2013).

46. Ebert, D., Faustov, R. N. \& Galkin, V. O. Properties of heavy quarkonia and $\mathrm{B}_{\mathrm{c}}$ mesons in the relativistic quark model. Phys. Rev. D 67, 014027 (2003)

47. Segovia, J. et al. Bottomonium spectrum revisited. Phys. Rev. D 93, 074027 (2016)

48. Cowan, G. et al. Asymptotic formulae for likelihood-based tests of new physics. Eur. Phys. J. C 71, 1554 (2011); erratum 73, 2501 (2013).

49. ATLAS Collaboration. Improved luminosity determination in pp collisions at $\sqrt{s}=7 \mathrm{TeV}$ using the ATLAS detector at the LHC. Eur. Phys. J. C 73, 2518 (2013).

50. ATLAS Collaboration. Luminosity determination in pp collisions at $\sqrt{s}=8 \mathrm{TeV}$ using the ATLAS detector at the LHC. Eur. Phys. J. C 76, 653 (2016).

51. ATLAS Collaboration. ATLAS Computing Acknowledgements 2016-2017, ATL-GEN-PUB-2016-002 (2016); https://cds.cern.ch/record/2199109

\section{Acknowledgements}

We thank CERN for the very successful operation of the LHC, as well as the support staff from our institutions without whom ATLAS could not be operated efficiently. We acknowledge the support of ANPCyT, Argentina; YerPhI, Armenia; ARC, Australia; BMWFW and FWF, Austria; ANAS, Azerbaijan; SSTC, Belarus; CNPq and FAPESP, Brazil; NSERC, NRC and CFI, Canada; CERN; CONICYT, Chile; CAS, MOST and NSFC, China; COLCIENCIAS, Colombia; MSMT CR, MPO CR and VSC CR, Czech Republic; DNRF and DNSRC, Denmark; IN2P3-CNRS, CEA-DSM/IRFU, France; GNSF, Georgia; BMBF, HGF and MPG, Germany; GSRT, Greece; RGC, Hong Kong SAR, China; ISF, I-CORE and Benoziyo Center, Israel; INFN, Italy; MEXT and JSPS, Japan; CNRST, Morocco; FOM and NWO, Netherlands; RCN, Norway; MNiSW and NCN, Poland; FCT, Portugal; MNE/IFA, Romania; MES of Russia and NRC KI, Russian Federation; JINR; MESTD, Serbia; MSSR, Slovakia; ARRS and MIZŠ, Slovenia; DST/NRF, South Africa; MINECO, Spain; SRC and Wallenberg Foundation, Sweden; SERI, SNSF and Cantons of Bern and Geneva, Switzerland; MOST, Taiwan; TAEK, Turkey; STFC, United Kingdom; DOE and NSF, United States of America. In addition, individual groups and members have received support from BCKDF, the Canada Council, CANARIE, CRC, Compute 
Canada, FQRNT, and the Ontario Innovation Trust, Canada; EPLANET, ERC, ERDF, FP7, Horizon 2020 and Marie Skłodowska-Curie Actions, European Union; Investissements d'Avenir Labex and Idex, ANR, Région Auvergne and Fondation Partager le Savoir, France; DFG and AvH Foundation, Germany; Herakleitos, Thales and Aristeia programmes co-financed by EU-ESF and the Greek NSRF; BSF, GIF and Minerva, Israel; BRF, Norway; CERCA Programme Generalitat de Catalunya, Generalitat Valenciana, Spain; the Royal Society and Leverhulme Trust, United Kingdom. The crucial computing support from all WLCG partners is acknowledged gratefully, in particular from CERN, the ATLAS Tier-1 facilities at TRIUMF (Canada), NDGF (Denmark, Norway, Sweden), CC-IN2P3 (France), KIT/GridKA (Germany), INFN-CNAF (Italy), NL-T1 (Netherlands), PIC (Spain), ASGC (Taiwan), RAL (UK) and BNL (USA), the Tier-2 facilities worldwide and large non-WLCG resource providers. Major contributors of computing resources are listed in ref. 51.

\section{Author contributions}

All authors have contributed to the publication, being variously involved in the design and the construction of the detectors, in writing software, calibrating subsystems, operating the detectors and acquiring data, and finally analysing the processed data. The ATLAS Collaboration members discussed and approved the scientific results. The manuscript was prepared by a subgroup of authors appointed by the collaboration and subject to an internal collaboration-wide review process. All authors reviewed and approved the final version of the manuscript.

\section{Additional information}

Supplementary information is available in the online version of the paper. Reprints and permissions information is available online at www.nature.com/reprints. Publisher's note: Springer Nature remains neutral with regard to jurisdictional claims in published maps and institutional affiliations. Correspondence and requests for materials should be addressed to ATLAS Collaboration.

\section{Competing financial interests}

The authors declare no competing financial interests.

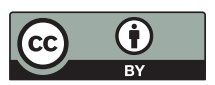

This article is licensed under a Creative Commons Attribution 4.0 International License, which permits use, sharing, adaptation, distribution and reproduction in any medium or format, as long as you give appropriate credit to the original author(s) and the source, provide a link to the Creative Commons license, and indicate if changes were made.

The images or other third party material in this article are included in the article's Creative Commons license, unless indicated otherwise in a credit line to the material. If material is not included in the article's Creative Commons license and your intended use is not permitted by statutory regulation or exceeds the permitted use, you will need to obtain permission directly from the copyright holder. To view a copy of this license, visit http://creativecommons.org/licenses/by/4.0/. 


\section{ATLAS Collaboration}

M. Aaboud ${ }^{181}$, G. Aad ${ }^{116}$, B. Abbott ${ }^{145}$, J. Abdallah' ${ }^{10}$, O. Abdinov ${ }^{14}$, B. Abeloos ${ }^{149}$, S. H. Abidi ${ }^{210}$, O. S. AbouZeid ${ }^{184}$, N. L. Abraham ${ }^{200}$, H. Abramowicz ${ }^{204}$, H. Abreu203, R. Abreu ${ }^{148}$, Y. Abulaiti ${ }^{196,197, ~ B . ~ S . ~ A c h a r y a ~}{ }^{218,219,236}$, S. Adachi' ${ }^{206}$, L. Adamczyk ${ }^{61}$, J. Adelman ${ }^{140}$, M. Adersberger ${ }^{131}$, T. Adye ${ }^{171}$, A. A. Affolder ${ }^{184}$, T. Agatonovic-Jovin ${ }^{16}$, C. Agheorghiesei ${ }^{39}$, J. A. Aguilar-Saavedra ${ }^{160,165}$, S. P. Ahlen ${ }^{30}$, F. Ahmadov ${ }^{95, b}$, G. Aielli ${ }^{174,175}$, S. Akatsuka ${ }^{98}$, H. Akerstedt ${ }^{196,197}$, T. P. A. Åkesson ${ }^{112}$, A. V. Akimov ${ }^{127}$, G. L. Alberghi ${ }^{27,28}$, J. Albert ${ }^{225}$, M. J. Alconada Verzini ${ }^{101}$, M. Aleksa ${ }^{46}$, I. N. Aleksandrov ${ }^{95}$, C. Alexa ${ }^{38}$, G. Alexander ${ }^{204}$, T. Alexopoulos ${ }^{12}$, M. Alhroob ${ }^{145}$, B. Ali ${ }^{168}$, M. Aliev ${ }^{103,104}$, G. Alimonti122, J. Alison ${ }^{47}$, S. P. Alkire ${ }^{57}$, B. M. M. Allbrooke ${ }^{200}$, B. W. Allen ${ }^{148}$, P. P. Allport ${ }^{21}$, A. Aloisio ${ }^{135,136}$, A. Alonso ${ }^{58}$, F. Alonso $^{101}$, C. Alpigiani ${ }^{185}$, A. A. Alshehri ${ }^{79}$, M. Alstaty ${ }^{116}$, B. Alvarez Gonzalez ${ }^{46}$, D. Álvarez Piqueras ${ }^{223}$, M. G. Alviggi ${ }^{135,136}$, B. T. Amadio ${ }^{18}$, Y. Amaral Coutinho ${ }^{32}$, C. Amelung ${ }^{31}$, D. Amidei ${ }^{120}$, S. P. Amor Dos Santos 160,162, A. Amorim ${ }^{160,161}$, S. Amoroso ${ }^{46}$, G. Amundsen ${ }^{31}$, C. Anastopoulos ${ }^{186}$ L. S. Ancu ${ }^{73}$, N. Andari ${ }^{21}$, T. Andeen ${ }^{13}$, C. F. Anders ${ }^{84}$, J. K. Anders ${ }^{105}$, K. J. Anderson 47, A. Andreazza ${ }^{122,123}$, V. Andrei ${ }^{83}$,

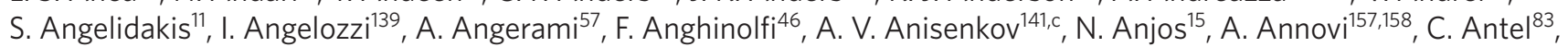
M. Antonelli ${ }^{71}$, A. Antonov ${ }^{129}$, D. J. Antrim ${ }^{217}$, F. Anulli172, M. Aoki ${ }^{96}$, L. Aperio Bella ${ }^{46}$, G. Arabidze ${ }^{121}$, Y. Arai ${ }^{96}$, J. P. Araque ${ }^{160}$, V. Araujo Ferraz ${ }^{32}$, A. T. H. Arce ${ }^{69}$, R. E. Ardell ${ }^{108}$, F. A. Arduh'101, J.-F. Arguin ${ }^{126}$, S. Argyropoulos ${ }^{93}$, M. Arik ${ }^{22}$, A. J. Armbruster ${ }^{190}$, L. J. Armitage ${ }^{107}$, O. Arnaez ${ }^{46}$, H. Arnold ${ }^{72}$, M. Arratia ${ }^{44}$, O. Arslan ${ }^{29}$, A. Artamonov ${ }^{128}$, G. Artoni ${ }^{152}$,

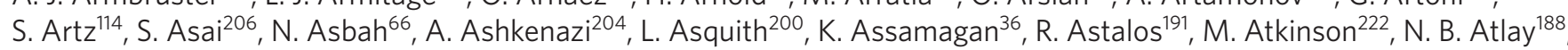
K. Augsten ${ }^{168}$, G. Avolio ${ }^{46}$, B. Axen ${ }^{18}$, M. K. Ayoub ${ }^{149}$, G. Azuelos ${ }^{126, d}$, A. E. Baas ${ }^{83}$, M. J. Baca ${ }^{21}$, H. Bachacou ${ }^{183}$, K. Bachas ${ }^{103,104}$, M. Backes ${ }^{152}$, M. Backhaus ${ }^{46}$, P. Bagiacchi ${ }^{172,173}$, P. Bagnaia ${ }^{172,173}$, J. T. Baines ${ }^{171}$, M. Bajic $^{58}$, O. K. Baker $^{232}$, E. M. Baldin ${ }^{141, c}$, P. Balek 228 , T. Balestri ${ }^{199}$, F. Balli ${ }^{183}$, W. K. Balunas ${ }^{155}$, E. Banas ${ }^{63}$, Sw. Banerjee 229, , A. A. E. Bannoura ${ }^{231}$, L. Barak ${ }^{46}$, E. L. Barberio ${ }^{119}$, D. Barberis ${ }^{74,75}$, M. Barbero ${ }^{116}$, T. Barillari ${ }^{132}$, M.-S. Barisits ${ }^{46}$, T. Barklow ${ }^{190}$, N. Barlow $^{44}$, S. L. Barnes ${ }^{55}$, B. M. Barnett ${ }^{171}$, R. M. Barnett ${ }^{18}$, Z. Barnovska-Blenessy ${ }^{53}$, A. Baroncelli ${ }^{176}$, G. Barone ${ }^{31}$, A. J. Barr ${ }^{152}$, L. Barranco Navarro ${ }^{223}$, F. Barreiro ${ }^{113}$, J. Barreiro Guimarães da Costa ${ }^{50}$, R. Bartoldus ${ }^{190}$, A. E. Barton ${ }^{102}$, P. Bartos ${ }^{191}$, A. Basalaev ${ }^{156}$, A. Bassalat149,, R. L. Bates ${ }^{79}$, S. J. Batista ${ }^{210}$, J. R. Batley ${ }^{44}$, M. Battaglia ${ }^{184}$, M. Bauce ${ }^{172,173}$, F. Bauer $^{183}$ H. S. Bawa ${ }^{190,8}$, J. B. Beacham43, M. D. Beattie ${ }^{102}$, T. Beau ${ }^{111}$, P. H. Beauchemin²16, P. Bechtle ${ }^{29}$, H. P. Beck ${ }^{20, h}, K^{2}$ Becker ${ }^{152}$, M. Becker ${ }^{114}$, M. Beckingham ${ }^{226}$, C. Becot ${ }^{142}$, A. J. Beddall25, A. Beddall23, V. A. Bednyakov ${ }^{95}$, M. Bedognetti ${ }^{139}$, C. P. Bee ${ }^{199}$, T. A. Beermann ${ }^{46}$, M. Begalli ${ }^{32}$, M. Begel ${ }^{36}$, J. K. Behr ${ }^{66}$, A. S. Bell ${ }^{109}$, G. Bella ${ }^{204}$, L. Bellagamba27, A. Bellerive ${ }^{45}$, M. Bellomo $^{117}$, K. Belotskiy ${ }^{129}$, O. Beltramello ${ }^{46}$, N. L. Belyaev ${ }^{129}$, O. Benary ${ }^{204}$, D. Benchekroun ${ }^{178}$, M. Bender ${ }^{131}$, K. Bendtz $^{196,197}$, N. Benekos $^{12}$, Y. Benhammou ${ }^{204}$, E. Benhar Noccioli' ${ }^{232}$, J. Benitez ${ }^{93}$, D. P. Benjamin ${ }^{69}$, M. Benoit ${ }^{73}$, J. R. Bensinger ${ }^{31}$, S. Bentvelsen ${ }^{139}$, L. Beresford ${ }^{152}$, M. Beretta ${ }^{71}$, D. Berge ${ }^{139}$, E. Bergeaas Kuutmann ${ }^{221}$, N. Berger ${ }^{7}$, J. Beringer ${ }^{18}$, S. Berlendis ${ }^{81}$, N. R. Bernard ${ }^{117}$, G. Bernardi ${ }^{111}$, C. Bernius ${ }^{142}$, F. U. Bernlochner ${ }^{29}$, T. Berry ${ }^{108}$, P. Berta ${ }^{169}$, C. Bertella ${ }^{114}$, G. Bertoli ${ }^{196,197}$, F. Bertolucci ${ }^{157,158}$, I. A. Bertram ${ }^{102}$, C. Bertsche 66 , D. Bertsche ${ }^{145}$, G. J. Besjes ${ }^{58}$, O. Bessidskaia Bylund ${ }^{196,197}$, M. Bessner ${ }^{66}$, N. Besson $^{183}$, C. Betancourt ${ }^{72}$, A. Bethani ${ }^{115}$, S. Bethke ${ }^{132}$, A. J. Bevan ${ }^{107}$, R. M. Bianchi ${ }^{159}$, M. Bianco ${ }^{46}$, O. Biebel ${ }^{131}$, D. Biedermann ${ }^{19}$, R. Bielski ${ }^{115}$, N. V. Biesuz ${ }^{157,158}$, M. Biglietti ${ }^{176}$, J. Bilbao De Mendizabal73, T. R. V. Billoud ${ }^{126}$, H. Bilokon $^{71}$, M. Bindi' ${ }^{80}$, A. Bingul ${ }^{23}$, C. Bini ${ }^{172,173}$, S. Biondi ${ }^{27,28}$, T. Bisanz ${ }^{80}$, C. Bittrich ${ }^{68}$, D. M. Bjergaard ${ }^{69}$, C. W. Black ${ }^{201}$, J. E. Black ${ }^{190}$, K. M. Black ${ }^{30}$, D. Blackburn ${ }^{185}$, R. E. Blair ${ }^{8}$, T. Blazek ${ }^{191}$, I. Bloch ${ }^{66}$, C. Blocker ${ }^{31}$, A. Blue ${ }^{79}$, W. Blum ${ }^{114}$, U. Blumenschein ${ }^{107}$, S. Blunier ${ }^{48}$, G. J. Bobbink ${ }^{139}$, V. S. Bobrovnikov ${ }^{141, c}$, S. S. Bocchetta ${ }^{112}$, A. Bocci ${ }^{69}$, C. Bock ${ }^{131}$, M. Boehler ${ }^{72}$, D. Boerner ${ }^{231}$, D. Bogavac ${ }^{131}$, A. G. Bogdanchikov ${ }^{141}$, C. Bohm ${ }^{196}$, V. Boisvert ${ }^{108}$, P. Bokan221,i T. Bold ${ }^{61}$, A. S. Boldyrev ${ }^{130}$, M. Bomben ${ }^{111}$, M. Bona ${ }^{107}$, M. Boonekamp ${ }^{183}$, A. Borisov ${ }^{170}$, G. Borissov ${ }^{102}$, J. Bortfeldt ${ }^{46}$, D. Bortoletto ${ }^{152}$, V. Bortolotto ${ }^{87,88,89}$, K. Bos $^{139}$, D. Boscherini ${ }^{27}$, M. Bosman ${ }^{15}$, J. D. Bossio Sola ${ }^{43}$, J. Boudreau ${ }^{159}$, J. Bouffard², E. V. Bouhova-Thacker ${ }^{102}$, D. Boumediene ${ }^{56}, C^{2}$ Bourdarios ${ }^{149}$, S. K. Boutle ${ }^{79}$, A. Boveia ${ }^{143}$, J. Boyd ${ }^{46}$, I. R. Boyko ${ }^{95}$, J. Bracinik ${ }^{21}$, A. Brandt ${ }^{10}$, G. Brandt ${ }^{80}$, O. Brandt ${ }^{83}$, U. Bratzler ${ }^{207}$, B. Brau ${ }^{117}$, J. E. Brau ${ }^{148}$, W. D. Breaden Madden ${ }^{79}$, K. Brendlinger ${ }^{66}$, A. J. Brennan ${ }^{119}$, L. Brenner ${ }^{139}$, R. Brenner ${ }^{221}$, S. Bressler ${ }^{228}$, D. L. Briglin' ${ }^{21}$, T. M. Bristow ${ }^{70}$, D. Britton ${ }^{79}$, D. Britzger ${ }^{66}$, F. M. Brochu ${ }^{44}$, I. Brock ${ }^{29}$, R. Brock ${ }^{121}$, G. Brooijmans ${ }^{57}$, T. Brooks ${ }^{108}$, W. K. Brooks ${ }^{49}$, J. Brosamer ${ }^{18}$, E. Brost ${ }^{140}$, J. H. Broughton ${ }^{21}$, P. A. Bruckman de Renstrom ${ }^{63}$, D. Bruncko ${ }^{192}$, A. Bruni ${ }^{27}$, G. Bruni ${ }^{27}$, L. S. Bruni ${ }^{139}$, B. H. Brunt ${ }^{44}$, M. Bruschi 27, N. Bruscino ${ }^{29}$, P. Bryant ${ }^{47}$, L. Bryngemark ${ }^{112}$, T. Buanes ${ }^{17}$, Q. Buat $^{189}$, P. Buchholz ${ }^{188}$, A. G. Buckley ${ }^{79}$, I. A. Budagov ${ }^{95}$, F. Buehrer ${ }^{72}$, M. K. Bugge ${ }^{151}$, O. Bulekov ${ }^{129}$, D. Bullock ${ }^{10}$, H. Burckhart ${ }^{46}$, S. Burdin ${ }^{105}$, C. D. Burgard ${ }^{72}$, A. M. Burger ${ }^{7}$, B. Burghgrave ${ }^{140}$, K. Burka ${ }^{63}$, S. Burke ${ }^{171}$, I. Burmeister ${ }^{67}$, J. T. P. Burr ${ }^{152}$, E. Busato ${ }^{56}$, D. Büscher ${ }^{72}$, V. Büscher ${ }^{114}$, P. Bussey ${ }^{79}$, J. M. Butler ${ }^{30}$, C. M. Buttar ${ }^{79}$, J. M. Butterworth ${ }^{109}$, P. Butti ${ }^{46}$, W. Buttinger ${ }^{36}$, A. Buzatu ${ }^{52}$, A. R. Buzykaev141,c , S. Cabrera Urbán ${ }^{223}$, D. Caforio ${ }^{168}$, V. M. Cairo ${ }^{59,60}$, O. Cakir ${ }^{4}$, N. Calace ${ }^{73}$, P. Calafiura ${ }^{18}$, A. Calandri ${ }^{116}$, G. Calderini ${ }^{111}$, P. Calfayan ${ }^{91}$, G. Callea ${ }^{59,60}$, L. P. Caloba ${ }^{32}$, S. Calvente Lopez ${ }^{113}$, D. Calvet ${ }^{56}$, S. Calvet ${ }^{56}$, T. P. Calvet ${ }^{116}$, R. Camacho Toro ${ }^{47}$, S. Camarda ${ }^{46}$, P. Camarri ${ }^{174,175}$, D. Cameron ${ }^{151}$, R. Caminal Armadans ${ }^{222}$, C. Camincher ${ }^{81}$, S. Campana ${ }^{46}$, M. Campanelli ${ }^{109}$, A. Camplani ${ }^{122,123}$, A. Campoverde ${ }^{188}$, V. Canale ${ }^{135,136}$, M. Cano Bret ${ }^{55}$, J. Cantero ${ }^{146}$, T. Cao ${ }^{204}$, M. D. M. Capeans Garrido ${ }^{46}$, I. Caprini ${ }^{38}$, M. Caprini ${ }^{38}$, M. Capua ${ }^{59,60}$, R. M. Carbone ${ }^{57}$, R. Cardarelli ${ }^{174}$, F. Cardillo ${ }^{72}$, I. Carli ${ }^{169}$, T. Carli ${ }^{46}$, G. Carlino ${ }^{135}$, B. T. Carlson ${ }^{159}$, L. Carminati ${ }^{122,123}$, R. M. D. Carney ${ }^{196,197}$, S. Caron ${ }^{138}$, E. Carquin ${ }^{49}$, G. D. Carrillo-Montoya ${ }^{46}$, J. Carvalho ${ }^{160,162}$, D. Casadei ${ }^{21}$, M. P. Casado ${ }^{15, j}$, M. Casolino ${ }^{15}$, D. W. Casper ${ }^{217}$, R. Castelijn ${ }^{139}$, A. Castelli ${ }^{139}$, V. Castillo Gimenez ${ }^{223}$, N. F. Castro ${ }^{160, k}$, A. Catinaccio ${ }^{46}$, J. R. Catmore ${ }^{151}$, A. Cattai ${ }^{46}$, J. Caudron ${ }^{29}$, V. Cavaliere ${ }^{222}$, E. Cavallaro ${ }^{15}$, D. Cavallii22, M. Cavalli-Sforza ${ }^{15}$, V. Cavasinni ${ }^{157,158}$, E. Celebi ${ }^{22}$, F. Ceradini ${ }^{176,177}$, L. Cerda Alberich ${ }^{223}$, A. S. Cerqueira ${ }^{33}$, A. Cerri ${ }^{200}$, L. Cerrito ${ }^{174,175}$, F. Cerutti ${ }^{18}$, A. Cervelli ${ }^{20}$, S. A. Cetin $^{24}$, A. Chafaq ${ }^{178}$, 
D. Chakraborty ${ }^{140}$, S. K. Chan ${ }^{82}$, W. S. Chan ${ }^{139}$, Y. L. Chan ${ }^{87}$, P. Chang ${ }^{222}$, J. D. Chapman ${ }^{44}$, D. G. Charlton ${ }^{21}$, A. Chatterjee ${ }^{73}$ C. C. Chau ${ }^{210}$, C. A. Chavez Barajas ${ }^{200}$, S. Che ${ }^{143}$, S. Cheatham ${ }^{218,220}$, A. Chegwidden"121, S. Chekanov ${ }^{8}$, S. V. Chekulaev ${ }^{213}$, G. A. Chelkovv5,I, M. A. Chelstowska ${ }^{46}$, C. Chen ${ }^{94}$, H. Chen ${ }^{36}$, S. Chen ${ }^{51}$, S. Chen ${ }^{206}$, X. Chen ${ }^{52, m}$, Y. Chen ${ }^{97}$, H. C. Cheng ${ }^{120,}$ H. J. Cheng ${ }^{50}$, Y. Cheng ${ }^{47}$, A. Cheplakov ${ }^{95}$, E. Cheremushkina ${ }^{170}$, R. Cherkaoui El Moursli ${ }^{182}$, V. Chernyatin ${ }^{36 \$}$, E. Cheu',

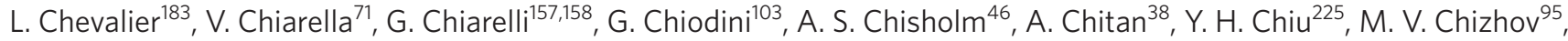
K. Choi ${ }^{91}$, A. R. Chomont ${ }^{56}$, S. Chouridou' ${ }^{11}$, B. K. B. Chow ${ }^{131}$, V. Christodoulou ${ }^{109}$, D. Chromek-Burckhart ${ }^{46}$, M. C. Chu ${ }^{87}$, J. Chudoba ${ }^{167}$, A. J. Chuinard ${ }^{118}$, J. J. Chwastowski ${ }^{63}$, L. Chytka ${ }^{147}$, A. K. Ciftci ${ }^{4}$, D. Cinca ${ }^{67}$, V. Cindro ${ }^{106}$, I. A. Cioara ${ }^{29}$, C. Ciocca ${ }^{27,28}$, A. Ciocio ${ }^{18}$, F. Cirotto ${ }^{135,136}$, Z. H. Citron ${ }^{228}$, M. Citterio ${ }^{122}$, M. Ciubancan ${ }^{38}$, A. Clark ${ }^{73}$, B. L. Clark ${ }^{82}$, M. R. Clark ${ }^{57}$, P. J. Clark ${ }^{70}$, R. N. Clarke ${ }^{18}$, C. Clement ${ }^{196,197}$, Y. Coadou ${ }^{116}$, M. Cobal ${ }^{218,220}$, A. Coccaro ${ }^{73}$, J. Cochran ${ }^{94}$, L. Colasurdo ${ }^{138}$, B. Cole ${ }^{57}$, A. P. Colijn ${ }^{139}$, J. Collot ${ }^{81}$, T. Colombo 217 , P. Conde Muiño ${ }^{160,161}$, E. Coniavitis ${ }^{72}$, S. H. Connell ${ }^{194}$, I. A. Connelly ${ }^{115}$, V. Consorti ${ }^{72}$, S. Constantinescu ${ }^{38}$, G. Conti ${ }^{46}$, F. Conventi ${ }^{135, n}$, M. Cooke ${ }^{18}$, B. D. Cooper ${ }^{109}$, A. M. Cooper-Sarkar ${ }^{152}$, F. Cormier 224 , K. J. R. Cormier ${ }^{210}$, T. Cornelissen ${ }^{231}$, M. Corradi ${ }^{172,173}$, F. Corriveau ${ }^{118,0}$, A. Cortes-Gonzalez ${ }^{46}$, G. Cortiana ${ }^{132}$, G. Costa ${ }^{122}$, M. J. Costa ${ }^{223}$, D. Costanzo ${ }^{186}$, G. Cottin ${ }^{44}$, G. Cowan ${ }^{108}$, B. E. Cox ${ }^{115}$, K. Cranmer $^{142}$, S. J. Crawley ${ }^{79}$, R. A. Creager ${ }^{155}$, G. Cree ${ }^{45}$, S. Crépé-Renaudin ${ }^{81}$, F. Crescioli111, W. A. Cribbs ${ }^{196,197}$, M. Crispin Ortuzar ${ }^{152}$, M. Cristinziani ${ }^{29}$, V. Croft ${ }^{138}$, G. Crosetti ${ }^{59,60}$, A. Cueto ${ }^{113}$, T. Cuhadar Donszelmann ${ }^{186}$, J. Cummings ${ }^{232}$, M. Curatolo ${ }^{71}$, J. Cúth ${ }^{114}$, H. Czirr ${ }^{188}$, P. Czodrowski ${ }^{46}$, G. D'amen ${ }^{27,28}$, S. D'Auria ${ }^{79}$, M. D'Onofrio ${ }^{105}$, M. J. Da Cunha Sargedas De Sousa ${ }^{160,161}$, C. Da Via ${ }^{115}$, W. Dabrowski61, T. Dado ${ }^{191}$, T. Dai ${ }^{120}$, O. Dale ${ }^{17}$, F. Dallaire ${ }^{126}$, C. Dallapiccola ${ }^{117}$, M. Dam ${ }^{58}$, J. R. Dandoy ${ }^{155}$, N. P. Dang ${ }^{72}$, A. C. Daniells ${ }^{21}$, N. S. Dann ${ }^{115}$, M. Danninger 224, M. Dano Hoffmann ${ }^{183}$, V. Dao ${ }^{199}$, G. Darbo ${ }^{74}$, S. Darmora ${ }^{10}$, J. Dassoulas ${ }^{3}$, A. Dattagupta ${ }^{148}$, T. Daubney ${ }^{66}$, W. Davey ${ }^{29}$, C. David ${ }^{66}$, T. Davidek ${ }^{169}$, M. Davies ${ }^{204}$, P. Davison ${ }^{109}$, E. Dawe ${ }^{119}$, I. Dawson ${ }^{186}$, K. De ${ }^{10}$, R. de Asmundis ${ }^{135}$, A. De Benedetti ${ }^{145}$, S. De Castro27,28, S. De Cecco ${ }^{111}$, N. De Groot ${ }^{138}$, P. de Jong ${ }^{139}$, H. De la Torre ${ }^{121}$, F. De Lorenzi ${ }^{94}$, A. De Maria ${ }^{80}$, D. De Pedis ${ }^{172}$, A. De Salvo ${ }^{172}$, U. De Sanctis ${ }^{174,175}$, A. De Santo ${ }^{200}$, K. De Vasconcelos Corga ${ }^{116}$ J. B. De Vivie De Regie ${ }^{149}$, W. J. Dearnaley ${ }^{102}$, R. Debbe ${ }^{36}$, C. Debenedetti ${ }^{184}$, D. V. Dedovich ${ }^{95}$, N. Dehghanian ${ }^{3}$, I. Deigaard ${ }^{139}$, M. Del Gaudio ${ }^{59,60}$, J. Del Peso ${ }^{113}$, T. Del Prete ${ }^{157,158}$, D. Delgove ${ }^{149}$, F. Deliot ${ }^{183}$, C. M. Delitzsch ${ }^{73}$, A. Dell'Acqua ${ }^{46}$, L. Dell'Asta ${ }^{30}$, M. Dell'Orso 157,158, M. Della Pietra ${ }^{135,136}$, D. della Volpe ${ }^{73}$, M. Delmastro ${ }^{7}$, C. Delporte ${ }^{149}$, P. A. Delsart ${ }^{81}$, D. A. DeMarco ${ }^{210}$, S. Demers ${ }^{232}$, M. Demichev ${ }^{95}$, A. Demilly ${ }^{111}$, S. P. Denisov ${ }^{170}$, D. Denysiuk ${ }^{183}$, D. Derendarz ${ }^{63}$, J. E. Derkaoui ${ }^{181}$, F. Derue ${ }^{111}$, P. Dervan ${ }^{105}$, K. Desch ${ }^{29}$, C. Deterre ${ }^{66}$, K. Dette ${ }^{67}$, P. O. Deviveiros ${ }^{46}$, A. Dewhurst ${ }^{171}$, S. Dhaliwal ${ }^{31}$, A. Di Ciaccio ${ }^{174,175}$, L. Di Ciaccio7, W. K. Di Clemente ${ }^{155}$, C. Di Donato ${ }^{135,136}$, A. Di Girolamo ${ }^{46}$, B. Di Girolamo ${ }^{46}$, B. Di Micco 176,177, R. Di Nardo 46, K. F. Di Petrillo82, A. Di Simone ${ }^{72}$, R. Di Sipio ${ }^{210}$, D. Di Valentino ${ }^{45}$, C. Diaconu ${ }^{116}$, M. Diamond ${ }^{210}$, F. A. Dias ${ }^{70}$, M. A. Diaz ${ }^{48}$, E. B. Diehl ${ }^{120}$, J. Dietrich ${ }^{19}$, S. Díez Cornell ${ }^{66}$, A. Dimitrievskaa ${ }^{16}$, J. Dingfelder ${ }^{29}$, P. Dita ${ }^{38}$, S. Dita ${ }^{38}$, F. Dittus ${ }^{46}$, F. Djama ${ }^{116}$, T. Djobava ${ }^{77}$, J. I. Djuvsland ${ }^{83}$, M. A. B. do Vale ${ }^{34}$, D. Dobos ${ }^{46}$, M. Dobre ${ }^{38}$, C. Doglioni ${ }^{112}$, J. Dolejsi ${ }^{169}$, Z. Dolezal ${ }^{169}$, M. Donadelli35, S. Donati157,158, P. Dondero ${ }^{153,154}$, J. Doninii ${ }^{56}$, J. Dopke ${ }^{171}$, A. Doria ${ }^{135}$, M. T. Dova ${ }^{101}$, A. T. Doyle ${ }^{79}$, E. Drechsler ${ }^{80}$, M. Dris ${ }^{12}$, Y. Du ${ }^{54}$, J. Duarte-Campderros ${ }^{204}$, E. Duchovni ${ }^{228}$, G. Duckeck ${ }^{131}$, A. Ducourthial111, O. A. Ducu ${ }^{126, \text { }}$, D. Duda ${ }^{139}$, A. Dudarev ${ }^{46}$, A. Chr. Dudder ${ }^{114}$, E. M. Duffield ${ }^{18}$, L. Duflot ${ }^{149}$, M. Dührssen ${ }^{46}$, M. Dumancic ${ }^{228}$, A. E. Dumitriu ${ }^{38}$, A. K. Duncan ${ }^{79}$, M. Dunford ${ }^{83}$, H. Duran Yildiz ${ }^{4}$, M. Düren ${ }^{78}$, A. Durglishvili ${ }^{77}$, D. Duschinger ${ }^{68}$, B. Dutta ${ }^{66}$, M. Dyndal ${ }^{66}$, C. Eckardt ${ }^{66}$, K. M. Ecker ${ }^{132}$, R. C. Edgar ${ }^{120}$, T. Eifert $^{46}$, G. Eigen ${ }^{17}$, K. Einsweiler $^{18}$, T. Ekelof ${ }^{221}$, M. El Kacimi ${ }^{180}$, R. El Kosseifi ${ }^{116}$, V. Ellajosyula ${ }^{116}$, M. Ellert ${ }^{221}$, S. Elles ${ }^{7}$, F. Ellinghaus ${ }^{231}$, A. A. Elliot ${ }^{225}$, N. Ellis $^{46}$, J. Elmsheuser ${ }^{36}$, M. Elsing ${ }^{46}$, D. Emeliyanov ${ }^{171}$, Y. Enari ${ }^{206}$, O. C. Endner ${ }^{114}$, J. S. Ennis ${ }^{226}$, J. Erdmann ${ }^{67}$, A. Ereditato ${ }^{20}$, G. Ernis ${ }^{231}$, M. Ernst ${ }^{36}$, S. Errede ${ }^{222}$, E. Ertel ${ }^{114}$, M. Escalier ${ }^{149}$, H. Esch ${ }^{67}$, C. Escobar ${ }^{159}$, B. Esposito ${ }^{71}$, A. I. Etienvre ${ }^{183}$,

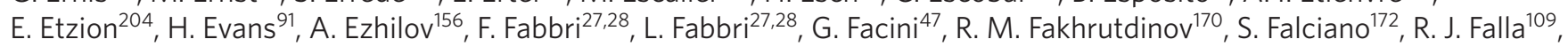
J. Faltova ${ }^{46}$, Y. Fang ${ }^{50}$, M. Fanti ${ }^{122,123}$, A. Farbin ${ }^{10}$, A. Farilla ${ }^{176}$, C. Farina ${ }^{159}$, E. M. Farina ${ }^{153,154}$, T. Farooque ${ }^{121}$, S. Farrell ${ }^{18}$, S. M. Farrington 226 , P. Farthouat ${ }^{46}$, F. Fassi ${ }^{182}$, P. Fassnacht ${ }^{46}$, D. Fassouliotis ${ }^{11}$, M. Faucci Giannelli108, A. Favareto ${ }^{74,75}$, W. J. Fawcett ${ }^{152}$, L. Fayard ${ }^{149}$, O. L. Fedin ${ }^{156,9}$, W. Fedorko 224 , S. Feigl ${ }^{151}$, L. Feligioni ${ }^{116}$, C. Feng ${ }^{54}$, E. J. Feng ${ }^{46}$, H. Feng ${ }^{120}$, A. B. Fenyuk ${ }^{170}$, L. Feremenga ${ }^{10}$, P. Fernandez Martinez ${ }^{223}$, S. Fernandez Perez ${ }^{15}$, J. Ferrando ${ }^{66}$, A. Ferrari' ${ }^{221}$, P. Ferrari ${ }^{139}$, R. Ferrari1 ${ }^{153}$, D. E. Ferreira de Lima ${ }^{84}$, A. Ferrer ${ }^{223}$, D. Ferrere ${ }^{73}$, C. Ferretti ${ }^{120}$, F. Fiedler ${ }^{114}$, A. Filipčic ${ }^{106}$, M. Filipuzzi ${ }^{66}$, F. Filthaut ${ }^{138}$, M. Fincke-Keeler ${ }^{225}$, K. D. Finelli201, M. C. N. Fiolhais ${ }^{160,162, r}$, L. Fiorini ${ }^{223}$, A. Fischer ${ }^{2}$, C. Fischer ${ }^{15}$, J. Fischer ${ }^{231}$, W. C. Fisher ${ }^{121}$, N. Flaschel ${ }^{66}$, I. Fleck ${ }^{188}$, P. Fleischmann ${ }^{120}$, R. R. M. Fletcher155, T. Flick ${ }^{231}$, B. M. Flierl ${ }^{131}$, L. R. Flores Castillo ${ }^{87}$, M. J. Flowerdew ${ }^{132}$, G. T. Forcolin ${ }^{115}$, A. Formica ${ }^{183}$, A. Forti1 ${ }^{115}$, A. G. Foster ${ }^{21}$, D. Fournier ${ }^{149}$, H. Fox ${ }^{102}$, S. Fracchia ${ }^{15}$,

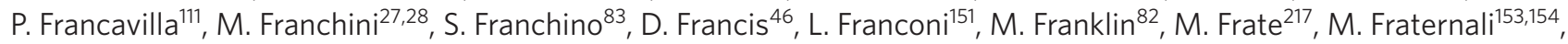
D. Freeborn ${ }^{109}$, S. M. Fressard-Batraneanu ${ }^{46}$, B. Freund ${ }^{126}$, D. Froidevaux ${ }^{46}$, J. A. Frost ${ }^{152}$, C. Fukunaga 207 ,

E. Fullana Torregrosa ${ }^{114}$, T. Fusayasu133, J. Fuster ${ }^{223}$, C. Gabaldon ${ }^{81}$, O. Gabizon ${ }^{203}$, A. Gabrielli27,28, A. Gabrielli ${ }^{18}$, G. P. Gach ${ }^{61}$, S. Gadatsch ${ }^{46}$, S. Gadomski ${ }^{108}$, G. Gagliardi ${ }^{74,75}$, L. G. Gagnon ${ }^{126}$, P. Gagnon ${ }^{91}$, C. Galea ${ }^{138}$, B. Galhardo 160,162, E. J. Gallas ${ }^{152}$, B. J. Gallop ${ }^{171}$, P. Gallus ${ }^{168}$, G. Galster ${ }^{58}$, K. K. Gan ${ }^{143}$, S. Ganguly ${ }^{56}$, J. Gao ${ }^{53}$, Y. Gao ${ }^{105}$, Y. S. Gao 190,8, F. M. Garay Walls ${ }^{70,}$ C. García ${ }^{223}$, J. E. García Navarro ${ }^{223}$, M. Garcia-Sciveres ${ }^{18}$, R. W. Gardner ${ }^{47}$, N. Garelli190, V. Garonne ${ }^{151}$, A. Gascon Bravo ${ }^{66}$, K. Gasnikova ${ }^{66}$, C. Gatti ${ }^{71}$, A. Gaudiello ${ }^{74,75}$, G. Gaudio ${ }^{153}$, I. L. Gavrilenko ${ }^{127}$, C. Gay ${ }^{224}$, G. Gaycken ${ }^{29}$, E. N. Gazis ${ }^{12}$, C. N. P. Gee ${ }^{171}$, M. Geisen ${ }^{114}$, M. P. Geisler ${ }^{83}$, K. Gellerstedt ${ }^{196,197}$, C. Gemme ${ }^{74}$, M. H. Genest ${ }^{81}$, C. Geng 53,5 , S. Gentile ${ }^{172,173}$, C. Gentsos ${ }^{205}$, S. George ${ }^{108}$, D. Gerbaudo ${ }^{15}$, A. Gershon ${ }^{204}$, S. Ghasemi ${ }^{188}$, M. Ghneimat ${ }^{29}$, B. Giacobbe ${ }^{27}$, S. Giagu ${ }^{172,173}$, P. Giannetti ${ }^{157,158}$, S. M. Gibson ${ }^{108}$, M. Gignac ${ }^{224}$, M. Gilchriese ${ }^{18}$, D. Gillberg ${ }^{45}$, G. Gilles ${ }^{231}$, D. M. Gingrich ${ }^{3, d}$, N. Giokaris ${ }^{11:}$, 


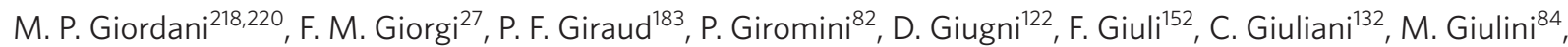
B. K. Gjelsten ${ }^{151}$, S. Gkaitatzis ${ }^{205}$, I. Gkialas ${ }^{11}$, E. L. Gkougkousis ${ }^{184}$, L. K. Gladilin ${ }^{130}$, C. Glasmann ${ }^{113}$, J. Glatzer ${ }^{15}$, P. C. F. Glaysher ${ }^{66}$, A. Glazov ${ }^{66}$, M. Goblirsch-Kolb ${ }^{31}$, J. Godlewski' ${ }^{63}$, S. Goldfarb ${ }^{119}$, T. Golling ${ }^{73}$, D. Golubkov ${ }^{170}$, A. Gomes ${ }^{160,161,163}$, R. Gonçalo ${ }^{160}$, R. Goncalves Gama ${ }^{32}$, J. Goncalves Pinto Firmino Da Costa ${ }^{183}$, G. Gonella ${ }^{72}$, L. Gonella21, A. Gongadze 95 , S. González de la Hoz ${ }^{223}$, S. Gonzalez-Sevilla ${ }^{73}$, L. Goossens ${ }^{46}$, P. A. Gorbounov ${ }^{128}$, H. A. Gordon ${ }^{36}$, I. Gorelov ${ }^{137}$, B. Gorini' ${ }^{46}$, E. Gorini103,104, A. Gorišek ${ }^{106}$, A. T. Goshaw ${ }^{69}$, C. Gössling ${ }^{67}$, M. I. Gostkin ${ }^{95}$, C. R. Goudet ${ }^{149}$, D. Goujdami ${ }^{180}$, A. G. Goussiou ${ }^{185}$, N. Govender ${ }^{194, t}$, E. Gozani ${ }^{203}$, L. Graber ${ }^{80}$, I. Grabowska-Bold ${ }^{61}$, P. O. J. Gradin 221, J. Gramling ${ }^{73}$, E. Gramstad ${ }^{151}$, S. Grancagnolo ${ }^{19}$, V. Gratchev ${ }^{156}$, P. M. Gravila ${ }^{42}$, H. M. Gray ${ }^{46}$, Z. D. Greenwood ${ }^{10,4}$, C. Grefe ${ }^{29}$, K. Gregersen ${ }^{109}$, I. M. Gregor ${ }^{66}$, P. Grenier ${ }^{190}$, K. Grevtsov ${ }^{7}$, J. Griffiths ${ }^{10}$, A. A. Grillo ${ }^{184}$, K. Grimm ${ }^{102}$, S. Grinstein ${ }^{15, v}$, Ph. Gris $^{56}$, J.-F. Grivaz ${ }^{149}$, S. Groh ${ }^{114}$, E. Gross ${ }^{228}$, J. Grosse-Knetter ${ }^{80}$, G. C. Grossi ${ }^{110}$, Z. J. Grout ${ }^{109}$, L. Guan ${ }^{120}$, W. Guan²29, J. Guenther ${ }^{92}$, F. Guescini213, D. Guest ${ }^{217}$, O. Gueta ${ }^{204}$, B. Guil43, E. Guido ${ }^{74,75}$, T. Guillemin 7 , S. Guindon², U. Gul ${ }^{79}$, C. Gumpert ${ }^{46}$, J. Guo $^{55}$, W. Guo ${ }^{120}$, Y. Guo ${ }^{53}$, R. Gupta ${ }^{64}$, S. Gupta ${ }^{152}$, G. Gustavino ${ }^{172,173}$, P. Gutierrez ${ }^{145}$, N. G. Gutierrez Ortiz ${ }^{109}$, C. Gutschow ${ }^{109}$, C. Guyot ${ }^{183}$, M. P. Guzik ${ }^{61}$, C. Gwenlan ${ }^{152}$, C. B. Gwilliam ${ }^{105}$, A. Haas ${ }^{142}$, C. Haber ${ }^{18}$, H. K. Hadavand ${ }^{10}$, A. Hadef ${ }^{116}$,

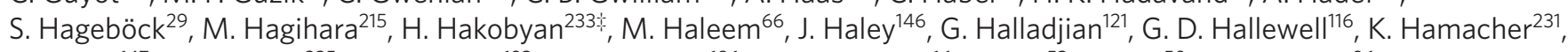
P. Hamal147, K. Hamano ${ }^{225}$, A. Hamilton ${ }^{193}$, G. N. Hamity ${ }^{186}$, P. G. Hamnett ${ }^{66}$, L. Han ${ }^{53}$, S. Han ${ }^{50}$, K. Hanagaki ${ }^{96, w}$,

K. Hanawa ${ }^{206}$, M. Hance ${ }^{184}$, B. Haney ${ }^{155}$, P. Hanke ${ }^{83}$, J. B. Hansen ${ }^{58}$, J. D. Hansen ${ }^{58}$, M. C. Hansen ${ }^{29}$, P. H. Hansen ${ }^{58}$, K. Hara ${ }^{215}$, A. S. Hard ${ }^{229}$, T. Harenberg ${ }^{231}$, F. Hariri ${ }^{149}$, S. Harkusha ${ }^{124}$, R. D. Harrington ${ }^{70}$, P. F. Harrison ${ }^{226}$, F. Hartjes ${ }^{139}$, N. M. Hartmann ${ }^{131^{\prime}}$, M. Hasegawa ${ }^{97}$, Y. Hasegawa ${ }^{187}$, A. Hasib ${ }^{70}$, S. Hassani ${ }^{183}$, S. Haug ${ }^{20}$, R. Hauser ${ }^{121}$, L. Hauswald ${ }^{68}$, L. B. Havener ${ }^{57}$, M. Havranek ${ }^{168}$, C. M. Hawkes ${ }^{21}$, R. J. Hawkings ${ }^{46}$, D. Hayakawa ${ }^{208}$, D. Hayden ${ }^{121}$, C. P. Hays ${ }^{152}$, J. M. Hays H. $^{107}$ H. S. Hayward'105, S. J. Haywood ${ }^{171}$, S. J. Head' ${ }^{21}$, T. Heck ${ }^{114}$, V. Hedberg ${ }^{112}$, L. Heelan ${ }^{10}$, K. K. Heidegger ${ }^{72}$, S. Heim ${ }^{66}$, T. Heim ${ }^{18}$, B. Heinemann ${ }^{66, x}$, J. J. Heinrich ${ }^{131}$, L. Heinrich ${ }^{142}$, C. Heinz ${ }^{78}$, J. Hejbal167, L. Helary ${ }^{46}$, A. Held ${ }^{224}$, S. Hellman ${ }^{196,197}$, C. Helsens ${ }^{46}$, J. Henderson ${ }^{152}$, R. C. W. Henderson ${ }^{102}$, Y. Heng ${ }^{229}$, S. Henkelmann ${ }^{224}$, A. M. Henriques Correia ${ }^{46}$, S. Henrot-Versille ${ }^{149}$, G. H. Herbert ${ }^{19}$, H. Herde ${ }^{31}$, V. Herget ${ }^{230}$, Y. Hernández Jiménez ${ }^{195}$, G. Herten ${ }^{72}$, R. Hertenberger ${ }^{131}$, L. Hervas ${ }^{46}$,

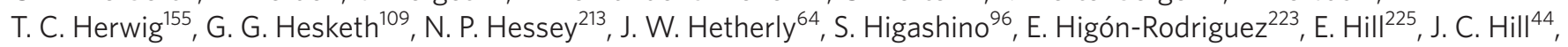
K. H. Hiller ${ }^{66}$, S. J. Hillier ${ }^{21}$, I. Hinchliffe ${ }^{18}$, M. Hirose ${ }^{72}$, D. Hirschbuehl ${ }^{231}$, B. Hiti106, O. Hladik ${ }^{167}$, X. Hoad ${ }^{10}$, J. Hobbs ${ }^{199}$, N. Hod ${ }^{213}$, M. C. Hodgkinson ${ }^{186}$, P. Hodgson ${ }^{186}$, A. Hoecker ${ }^{46}$, M. R. Hoeferkamp ${ }^{137}$, F. Hoenig' ${ }^{131}$, D. Hohn ${ }^{29}$, T. R. Holmes ${ }^{18}$, M. Homann ${ }^{67}$, S. Honda 215 , T. Honda ${ }^{96}$, T. M. Hong ${ }^{159}$, B. H. Hooberman²22, W. H. Hopkins ${ }^{148}$, Y. Horii ${ }^{134}$, A. J. Horton ${ }^{189}$, J.-Y. Hostachy" ${ }^{81}$, S. Hou ${ }^{202}$, A. Hoummada ${ }^{178}$, J. Howarth ${ }^{66}$, J. Hoya ${ }^{101}$, M. Hrabovsky ${ }^{147}$, I. Hristova ${ }^{19}$, J. Hrivnac ${ }^{149}$,

T. Hryn'ova7, A. Hrynevich ${ }^{125}$, P. J. Hsu ${ }^{90}$, S.-C. Hsu' ${ }^{185}$, Q. Hu ${ }^{53}$, S. Hu ${ }^{55}$, Y. Huang ${ }^{50}$, Z. Hubacek ${ }^{168}$, F. Hubaut ${ }^{116}$, F. Huegging ${ }^{29}$, T. B. Huffman ${ }^{152}$, E. W. Hughes ${ }^{57}$, G. Hughes ${ }^{102}$, M. Huhtinen ${ }^{46}$, P. Huo ${ }^{199}$, N. Huseynov ${ }^{95,6}$, J. Huston ${ }^{121}$, J. Huth ${ }^{82}$, G. lacobucci ${ }^{73}$, G. lakovidis ${ }^{36}$, I. Ibragimov ${ }^{188}$, L. Iconomidou-Fayard ${ }^{149}$, P. lengo ${ }^{46}$, O. Igonkina ${ }^{139, y}$, T. lizawa ${ }^{227}$, Y. Ikegami $^{96}$, M. Ikeno ${ }^{96}$, Y. Ilchenko ${ }^{13,2}$, D. Iliadis ${ }^{205}$, N. Ilic ${ }^{190}$, G. Introzzi ${ }^{153,154}$, P. Ioannou ${ }^{11 \div}$, M. Iodice ${ }^{176}$, K. Iordanidou ${ }^{57}$, V. Ippolito ${ }^{82}$, N. Ishijima ${ }^{150}$, M. Ishino ${ }^{206}$, M. Ishitsuka ${ }^{208}$, C. Issever ${ }^{152}$, S. Istin ${ }^{22}$, F. Ito ${ }^{215}$, J. M. Iturbe Ponce ${ }^{115}$, R. Iuppa ${ }^{211,212}$, H. Iwasaki ${ }^{96}$,

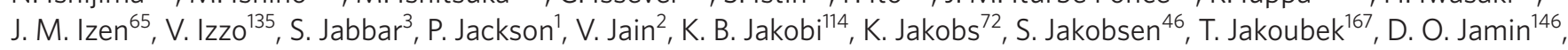
D. K. Jana ${ }^{110}$, R. Jansky ${ }^{92}$, J. Janssen ${ }^{29}$, M. Janus ${ }^{80}$, P. A. Janus ${ }^{61}$, G. Jarlskog ${ }^{112}$, N. Javadov ${ }^{95,6}$, T. Javůrek ${ }^{72}$, M. Javurkova ${ }^{72}$, F. Jeanneau ${ }^{183}$, L. Jeanty ${ }^{18}$, J. Jejelava ${ }^{76, a a}$, A. Jelinskas ${ }^{226}$, P. Jenni ${ }^{72, a b}$, C. Jeske ${ }^{226}$, S. Jézéquel ${ }^{7}$, H. Ji229, J. Jia ${ }^{199}$, H. Jiang ${ }^{94}$, Y. Jiang ${ }^{53}$, Z. Jiang ${ }^{190}$, S. Jiggins ${ }^{109}$, J. Jimenez Pena ${ }^{223}$, S. Jin ${ }^{50}$, A. Jinaru ${ }^{38}$, O. Jinnouchi ${ }^{208}$, H. Jivan ${ }^{195}$, P. Johansson ${ }^{186}$, K. A. Johns ${ }^{9}$, C. A. Johnson ${ }^{91}$, W. J. Johnson ${ }^{185}$, K. Jon-And ${ }^{196,197}$, R. W. L. Jones ${ }^{102}$, S. Jones ${ }^{9}$, T. J. Jones ${ }^{105}$, J. Jongmanns ${ }^{83}$, P. M. Jorge ${ }^{160,161}$, J. Jovicevic ${ }^{213}$, X. Ju229, A. Juste Rozas ${ }^{15, v}$, M. K. Köhler ${ }^{228}$, A. Kaczmarska ${ }^{63}$, M. Kado ${ }^{149}$, H. Kagan ${ }^{143}$, M. Kagan ${ }^{190}$, S. J. Kahn ${ }^{116}$, T. Kaji227, E. Kajomovitz ${ }^{69}$, C. W. Kalderon ${ }^{112}$, A. Kaluza914, S. Kama ${ }^{64}$, A. Kamenshchikov ${ }^{170}$, N. Kanaya206, S. Kaneti ${ }^{44}$, L. Kanjir ${ }^{106}$, V. A. Kantserov ${ }^{129}$, J. Kanzaki ${ }^{96}$, B. Kaplan ${ }^{122}$, L. S. Kaplan²29, D. Kar ${ }^{195}$, K. Karakostas $^{12}$, N. Karastathis ${ }^{12}$, M. J. Kareem ${ }^{80}$, E. Karentzos ${ }^{12}$, S. N. Karpov95, Z. M. Karpova95, K. Karthik ${ }^{142}$, V. Kartvelishvili"102, A. N. Karyukhin ${ }^{170}$, K. Kasahara ${ }^{215}$, L. Kashif 229 , R. D. Kass ${ }^{143}$, A. Kastanas ${ }^{198}$, Y. Kataoka ${ }^{206}$, C. Kato ${ }^{206}$, A. Katre ${ }^{73}$, J. Katzy ${ }^{66}$, K. Kawade ${ }^{134}$, K. Kawagoe ${ }^{100}$, T. Kawamoto ${ }^{206}$, G. Kawamura ${ }^{80}$, E. F. Kay ${ }^{105}$, V. F. Kazanin ${ }^{141, c}$, R. Keeler ${ }^{225}$, R. Kehoe ${ }^{64}$, J. S. Keller ${ }^{66}$, J. J. Kempster ${ }^{108}$, Keoshkerian ${ }^{210}$, O. Kepka ${ }^{167}$, B. P. Kerševan ${ }^{106}$, S. Kersten ${ }^{231}$, R. A. Keyes ${ }^{118}$, M. Khader ${ }^{222}$ F. Khalil-zada ${ }^{14}$, A. Khanov ${ }^{146}$, A. G. Kharlamov ${ }^{141, c}$, T. Kharlamova ${ }^{141, c}$, A. Khodinov209, T. J. Khoo ${ }^{73}$, V. Khovanskiy ${ }^{128:}$, E. Khramov ${ }^{95}$, J. Khubua ${ }^{77, a c}$, S. Kido ${ }^{97}$, C. R. Kilby ${ }^{108}$, H. Y. Kim¹0, S. H. Kim²15, Y. K. Kim ${ }^{47}$, N. Kimura ${ }^{205}$, O. M. Kind ${ }^{19}$, B. T. King ${ }^{105}$, D. Kirchmeier ${ }^{68}$, J. Kirk ${ }^{171}$, A. E. Kiryunin ${ }^{132}$, T. Kishimoto ${ }^{206}$, D. Kisielewska ${ }^{61}$, K. Kiuchi2 ${ }^{215}$, O. Kivernyk E. Kladiva ${ }^{192}$, T. Klapdor-Kleingrothaus ${ }^{72}$, M. H. Klein ${ }^{57}$, M. Klein ${ }^{105}$, U. Klein ${ }^{105}$, K. Kleinknecht $^{114}$, P. Klimek ${ }^{140}$, A. Klimentov ${ }^{36}$, R. Klingenberg ${ }^{67}$, T. Klingl ${ }^{29}$, T. Klioutchnikova ${ }^{46}$, E.-E. Kluge ${ }^{83}$, P. Kluit ${ }^{139}$, S. Kluth ${ }^{132}$, J. Knapik ${ }^{63}$, E. Kneringer ${ }^{92}$, E. B. F. G. Knoops ${ }^{116}$, A. Knue ${ }^{132}$, A. Kobayashi ${ }^{206}$, D. Kobayashi ${ }^{208}$, T. Kobayashi ${ }^{206}$, M. Kobel ${ }^{68}$, M. Kocian ${ }^{190}$, P. Kodys ${ }^{169}$, T. Koffas ${ }^{45}$, E. Koffeman ${ }^{139}$, N. M. Köhler ${ }^{132}$, T. Koi ${ }^{190}$, M. Kolb ${ }^{84}$, I. Koletsou7 , A. A. Komar ${ }^{127}$, Y. Komori ${ }^{206}$, T. Kondo ${ }^{96}$, N. Kondrashova ${ }^{55}$, K. Köneke ${ }^{72}$, A. C. König ${ }^{138}$, T. Kono ${ }^{96, a d}$, R. Konoplich ${ }^{142, a e}$, N. Konstantinidis ${ }^{109}$, R. Kopeliansky ${ }^{91}$,

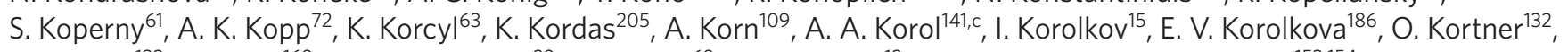
S. Kortner ${ }^{132}$, T. Kosek ${ }^{169}$, V. V. Kostyukhin ${ }^{29}$, A. Kotwal ${ }^{69}$, A. Koulouris ${ }^{12}$, A. Kourkoumeli-Charalampidi 153,154, C. Kourkoumelis" ${ }^{11}$, E. Kourlitis ${ }^{186}$, V. Kouskoura ${ }^{36}$, A. B. Kowalewska ${ }^{63}$, R. Kowalewski ${ }^{225}$, T. Z. Kowalski' ${ }^{61}$, C. Kozakai ${ }^{206}$, W. Kozanecki ${ }^{183}$, A. S. Kozhin ${ }^{170}$, V. A. Kramarenko ${ }^{130}$, G. Kramberger ${ }^{106}$, D. Krasnopevtsev ${ }^{129}$, A. Krasznahorkay ${ }^{46}$, 
D. Krauss ${ }^{132}$, A. Kravchenko ${ }^{36}$, J. A. Kremer ${ }^{61}$, M. Kretz ${ }^{85}$, J. Kretzschmar ${ }^{105}$, K. Kreutzfeldt ${ }^{78}$, P. Krieger ${ }^{210}, K^{2}$ Krizka ${ }^{47}$, K. Kroeninger ${ }^{67}$, H. Kroha ${ }^{132}$, J. Kroll ${ }^{155}$, J. Kroseberg ${ }^{29}$, J. Krstic ${ }^{16}$, U. Kruchonak ${ }^{95}$, H. Krüger ${ }^{29}$, N. Krumnack ${ }^{94}$, M. C. Kruse ${ }^{69}$, M. Kruskal ${ }^{30}$, T. Kubota ${ }^{119}$, H. Kucuk ${ }^{109}$, S. Kuday ${ }^{5}$, J. T. Kuechler ${ }^{231}$, S. Kuehn ${ }^{46}$, A. Kugel ${ }^{85}$, F. Kuger230, T. Kuhl66, V. Kukhtin ${ }^{95}$, R. Kukla ${ }^{116}$, Y. Kulchitsky ${ }^{124}$, S. Kuleshov ${ }^{49}$, Y. P. Kulinich ${ }^{222}$, M. Kuna ${ }^{172,173}$, T. Kunigo ${ }^{98}$, A. Kupco ${ }^{167}$, O. Kuprash ${ }^{204}$ H. Kurashige ${ }^{97}$, L. L. Kurchaninov ${ }^{213}$, Y. A. Kurochkin ${ }^{124}$, M. G. Kurth ${ }^{50}$, V. Kus ${ }^{167}$, E. S. Kuwertz ${ }^{225}$, M. Kuze ${ }^{208}$, J. Kvitaa ${ }^{147}$, T. Kwan ${ }^{225}$, D. Kyriazopoulos ${ }^{186}$, A. La Rosa ${ }^{132}$, J. L. La Rosa Navarro ${ }^{35}$, L. La Rotonda ${ }^{59,60}$, C. Lacasta ${ }^{223}$, F. Lacava $a^{172,173, ~}$ J. Lacey ${ }^{66}$, H. Lacker ${ }^{19}$, D. Lacour ${ }^{111}$, E. Ladygin ${ }^{95}$, R. Lafaye7, B. Laforge ${ }^{111}$, T. Lagouri232, S. Lai ${ }^{80}$, S. Lammers ${ }^{91}$, W. Lampl9, E. Lançon ${ }^{36}$, U. Landgraf ${ }^{72}$, M. P. J. Landon ${ }^{107}$, M. C. Lanfermann ${ }^{73}$, V. S. Lang83 , J. C. Lange ${ }^{15}$, A. J. Lankford ${ }^{217}$, F. Lanni ${ }^{36}$, K. Lantzsch ${ }^{29}$, A. Lanza ${ }^{153}$, A. Lapertosa ${ }^{74,75}$, S. Laplace ${ }^{111}$, J. F. Laporte ${ }^{183}$, T. Lari ${ }^{122}$, F. Lasagni Manghi ${ }^{27,28}$, M. Lassnig ${ }^{46}$, P. Laurelli ${ }^{71}$, W. Lavrijsen ${ }^{18}$, A. T. Law ${ }^{184}$, P. Laycock ${ }^{105}$, T. Lazovich ${ }^{82}$, M. Lazzaroni ${ }^{122,123}$, B. Le ${ }^{119}$, O. Le Dortz ${ }^{111}$, E. Le Guirriec ${ }^{116}$, E. P. Le Quilleuc ${ }^{183}$, M. LeBlanc ${ }^{225}$, T. LeCompte ${ }^{8}$, F. Ledroit-Guillon ${ }^{81}$, C. A. Lee ${ }^{36}$, S. C. Lee ${ }^{202}$, L. Lee', B. Lefebvre ${ }^{118}$, G. Lefebvre ${ }^{111}$, M. Lefebvre ${ }^{225}$, F. Legger ${ }^{131}$, C. Leggett ${ }^{18}$, A. Lehan ${ }^{105}$, G. Lehmann Miotto ${ }^{46}$, X. Lei ${ }^{9}$, W. A. Leight ${ }^{66}$, M. A. L. Leite ${ }^{35}$, R. Leitner ${ }^{169}$, D. Lellouch ${ }^{228}$, B. Lemmer ${ }^{80}$, K. J. C. Leney ${ }^{109}$, T. Lenz ${ }^{29}$, B. Lenzi ${ }^{46}$, R. Leone' ${ }^{9}$ S. Leone ${ }^{157,158,}$ C. Leonidopoulos ${ }^{70}$, G. Lerner ${ }^{200}$, C. Leroy ${ }^{126}$, A. A. J. Lesage ${ }^{183}$, C. G. Lester ${ }^{44}$, M. Levchenko ${ }^{156}$, J. Levêque ${ }^{7}$, D. Levin ${ }^{120}$,

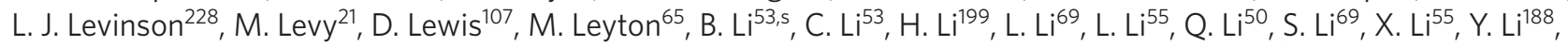

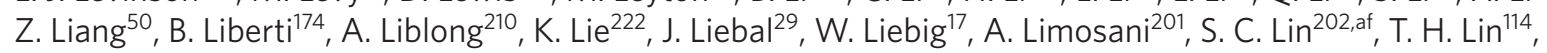
B. E. Lindquist ${ }^{199}$, A. E. Lionti ${ }^{73}$, E. Lipeles ${ }^{155}$, A. Lipniacka ${ }^{17}$, M. Lisovyi ${ }^{84}$, T. M. Liss ${ }^{222}$, A. Lister ${ }^{224}$, A. M. Litke ${ }^{184}$, B. Liu 202,ag,

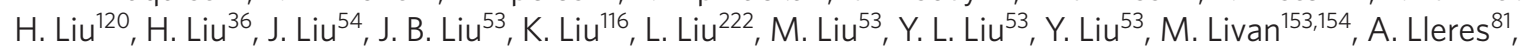
J. Llorente Merino ${ }^{50}$, S. L. Lloyd ${ }^{107}$, C. Y. Lo ${ }^{88}$, F. Lo Sterzo ${ }^{202}$, E. M. Lobodzinska ${ }^{66}$, P. Loch ${ }^{9}$, F. K. Loebinger ${ }^{115}$, K. M. Loew ${ }^{31}$, A. Loginov ${ }^{232}$, T. Lohse ${ }^{19}$, K. Lohwasser ${ }^{66}$, M. Lokajicek ${ }^{167}$, B. A. Long ${ }^{30}$, J. D. Long ${ }^{222}$, R. E. Long ${ }^{102}$, L. Longo ${ }^{103,104}$, K. A. Looper ${ }^{143}$, J. A. Lopez ${ }^{49}$, D. Lopez Mateos ${ }^{82}$, I. Lopez Paz ${ }^{15}$, A. Lopez Solis ${ }^{111}$, J. Lorenz ${ }^{131}$, N. Lorenzo Martinez

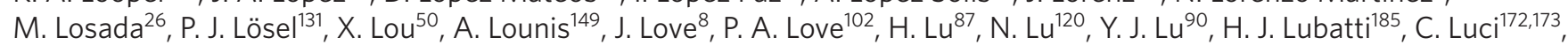
A. Lucotte ${ }^{81}$, C. Luedtke ${ }^{72}$, F. Luehring ${ }^{91}$, W. Lukas ${ }^{92}$, L. Luminari172, O. Lundberg96,197, B. Lund-Jensen ${ }^{198}$, P. M. Luzi ${ }^{111}$, D. Lynn ${ }^{36}$, R. Lysak'167, E. Lytken ${ }^{112}$, V. Lyubushkinn ${ }^{95}$, H. Ma ${ }^{36}$, L. L. Ma ${ }^{54}$, Y. Ma ${ }^{54}$, G. Maccarrone ${ }^{71}$, A. Macchiolo ${ }^{132}$, C. M. Macdonald ${ }^{186}$, B. Maček ${ }^{106}$, J. Machado Miguens ${ }^{155,161}$, D. Madaffari ${ }^{116}$, R. Madar ${ }^{56}$, H. J. Maddocks ${ }^{221}$, W. F. Mader ${ }^{68}$, A. Madsen ${ }^{66}$, J. Maeda ${ }^{97}$, S. Maeland ${ }^{17}$, T. Maeno ${ }^{36}$, A. Maevskiy ${ }^{130}$, E. Magradze ${ }^{80}$, J. Mahlstedt ${ }^{139}$, C. Maiani ${ }^{149}$,

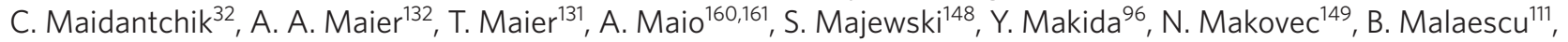
Pa. Malecki63, V. P. Maleev ${ }^{156}$, F. Malek ${ }^{81}$, U. Mallik ${ }^{93}$, D. Malon ${ }^{8}$, C. Malone ${ }^{44}$, S. Maltezos ${ }^{12}$, S. Malyukov ${ }^{46}$, J. Mamuzic ${ }^{223}$, G. Mancini ${ }^{11}$, L. Mandelli ${ }^{122}$, I. Mandić106, J. Maneira ${ }^{160,161}$, L. Manhaes de Andrade Filho ${ }^{33}$, J. Manjarres Ramos ${ }^{214}$, A. Mann ${ }^{131}$, A. Manousos ${ }^{46}$, B. Mansoulie ${ }^{183}$, J. D. Mansour ${ }^{50}$, R. Mantifel ${ }^{118}$, M. Mantoaniio, S. Manzoni ${ }^{122,123}$, L. Mapelli46, G. Marceca ${ }^{43}$, L. March ${ }^{73}$, G. Marchiori ${ }^{111}$, M. Marcisovsky ${ }^{167}$, M. Marjanovic ${ }^{56}$, D. E. Marley ${ }^{120}$, F. Marroquim², S. P. Marsden ${ }^{115}$, Z. Marshall18, M. U. F. Martensson 221, S. Marti-Garcia223, C. B. Martinn"13, T. A. Martin226, V. J. Martin ${ }^{70}$, B. Martin dit Latour ${ }^{17}$, M. Martinez ${ }^{15, v}$, V. I. Martinez Outschoornn 222 , S. Martin-Haugh ${ }^{171}$, V. S. Martoiu ${ }^{38}$, A. C. Martyniuk ${ }^{109}$, A. Marzin ${ }^{145}$, L. Masetti14, T. Mashimo 206 , R. Mashinistov ${ }^{127}$, J. Masik ${ }^{115}$, A. L. Maslennikov ${ }^{141, c}$, L. Massa ${ }^{174,175}$, P. Mastrandrea7, A. Mastroberardino ${ }^{59,60}$, T. Masubuchi206, P. Mättig231, J. Maurer ${ }^{38}$, S. J. Maxfield ${ }^{105}$, D. A. Maximov141,c, R. Mazini ${ }^{202}$, I. Maznas ${ }^{205}$, S. M. Mazza ${ }^{122,123}$, N. C. Mc Fadden"137, G. McGoldrick²0, S. P. McKee ${ }^{120}$, A. McCarn ${ }^{120}$, R. L. McCarthy' ${ }^{199}$, T. G. McCarthy ${ }^{132}$, L. I. McClymont ${ }^{109}$, E. F. McDonald ${ }^{119}$, J. A. Mcfayden ${ }^{109}$, G. Mchedlidze ${ }^{80}$, S. J. McMahon ${ }^{171}$, P. C. McNamara ${ }^{119}$, R. A. McPherson 225,0 , S. Meehan ${ }^{185}$, T. J. Megy ${ }^{72}$, S. Mehlhase ${ }^{131}$, A. Mehta ${ }^{105}$, T. Meideck ${ }^{81}, K^{2}$. Meier ${ }^{83}$, C. Meineck'131, B. Meirose ${ }^{65}$, D. Melini223,ah, B. R. Mellado Garcia ${ }^{195}$, M. Meloo ${ }^{191}$, F. Meloni ${ }^{20}$, S. B. Menary ${ }^{115}$, L. Meng ${ }^{105}$, X. T. Meng ${ }^{120}$, A. Mengarelli27,28, S. Menke ${ }^{132}$, E. Meoni ${ }^{216}$, S. Mergelmeyer ${ }^{19}$, P. Mermod ${ }^{73}$, L. Merola ${ }^{135,136}$, C. Meroni $^{122}$, F. S. Merritt ${ }^{47}$, A. Messina ${ }^{172,173}$, J. Metcalfe ${ }^{8}$, A. S. Mete ${ }^{217}$, C. Meyer ${ }^{155}$, J.-P. Meyer ${ }^{183}$, J. Meyer ${ }^{139}$, H. Meyer Zu Theenhausen ${ }^{83}$, F. Miano ${ }^{200}$, R. P. Middleton ${ }^{171}$, S. Miglioranzi ${ }^{74,75}$, L. Mijović ${ }^{70}$, G. Mikenberg 228 , M. Mikestikova ${ }^{167}$, M. Mikuž ${ }^{106}$, M. Milesi ${ }^{119}$, A. Milic ${ }^{36}$, D. W. Miller ${ }^{47}$, C. Mills ${ }^{70}$, A. Milov ${ }^{228}$, D. A. Milstead ${ }^{196,197}$, A. A. Minaenko ${ }^{170}$, Y. Minami206, I. A. Minashvili" ${ }^{95}$, A. I. Mincer ${ }^{142}$, B. Mindur ${ }^{61}$, M. Mineev ${ }^{95}$, Y. Minegishi206, Y. Ming ${ }^{229}$, L. M. Mir ${ }^{15}$, K. P. Mistry ${ }^{155}$, T. Mitani ${ }^{227}$, J. Mitrevski131, V. A. Mitsou 223 , A. Miucci ${ }^{20}$, P. S. Miyagawa ${ }^{186}$, A. Mizukami ${ }^{96}$, J. U. Mjörnmark ${ }^{12}$, M. Mlynarikova169, T. Moa ${ }^{196,197}$, K. Mochizuki26, P. Mogg72, S. Mohapatra ${ }^{57}$, S. Molander ${ }^{196,197}$, R. Moles-Valls ${ }^{29}$, R. Monden ${ }^{98}$, M. C. Mondragon ${ }^{121}$, K. Mönig ${ }^{66}$, J. Monk ${ }^{58}$, E. Monnier ${ }^{116}$, A. Montalbano ${ }^{199}$, J. Montejo Berlingen ${ }^{46}$, F. Monticelli101, S. Monzani ${ }^{122,123}$, R. W. Moore ${ }^{3}$, N. Morange ${ }^{149}$, D. Moreno ${ }^{26}$, M. Moreno Llácer ${ }^{80}$, P. Morettini ${ }^{74}$, S. Morgenstern ${ }^{46}$, D. Mori ${ }^{189}$, T. Mori ${ }^{206}$, M. Morii ${ }^{82}$, M. Morinagaa ${ }^{206}$, V. Morisbak ${ }^{151}$, A. K. Morley ${ }^{201}$, G. Mornacchi' ${ }^{46}$, J. D. Morris ${ }^{107}$, L. Morvaj ${ }^{199}$, P. Moschovakos ${ }^{12}$, M. Mosidze ${ }^{77}$, H. J. Moss' ${ }^{186}$, J. Moss 190, ai , K. Motohashi208, R. Mount ${ }^{190}$, E. Mountricha ${ }^{36}$, E. J. W. Moyse ${ }^{117}$, S. Muanza ${ }^{116}$, R. D. Mudd ${ }^{21}$, F. Mueller ${ }^{132}$, J. Mueller ${ }^{159}$, R. S. P. Mueller ${ }^{131}$, D. Muenstermann ${ }^{102}$, P. Mullen ${ }^{79}$, G. A. Mullier ${ }^{20}$, F. J. Munoz Sanchez ${ }^{115}$, W. J. Murray ${ }^{226,171}$, H. Musheghyan ${ }^{80}$,

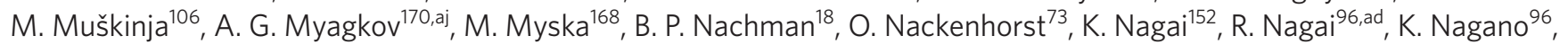
Y. Nagasaka ${ }^{86}$, K. Nagataa ${ }^{215}$, M. Nagel ${ }^{72}$, E. Nagy ${ }^{116}$, A. M. Nairz ${ }^{46}$, Y. Nakahama ${ }^{134}$, K. Nakamura ${ }^{66}$, T. Nakamura ${ }^{206}$, I. Nakano ${ }^{144}$, R. F. Naranjo Garcia ${ }^{66}$, R. Narayann ${ }^{13}$, D. I. Narrias Villar ${ }^{83}$, I. Naryshkin ${ }^{156}$, T. Naumann ${ }^{66}$, G. Navarro ${ }^{26}$, R. Nayyar ${ }^{9}$, H. A. Neal ${ }^{120}$, P. Yu. Nechaeva ${ }^{127}$, T. J. Neep ${ }^{183}$, A. Negri ${ }^{153,154}$, M. Negrini2 ${ }^{27}$, S. Nektarijevic ${ }^{138}$, C. Nellist ${ }^{149}$, A. Nelson ${ }^{217}$, M. E. Nelson ${ }^{152}$, S. Nemecek ${ }^{167}$, P. Nemethy ${ }^{142}$, A. A. Nepomuceno ${ }^{32}$, M. Nessi ${ }^{46, a k}$, M. S. Neubauer ${ }^{222}$, 
M. Neumann ${ }^{231}$, R. M. Neves ${ }^{142}$, P. R. Newman ${ }^{21}$, T. Y. Ng ${ }^{89}$, T. Nguyen Manh ${ }^{126}$, R. B. Nickerson ${ }^{152}$, R. Nicolaidou ${ }^{183}$ J. Nielsen ${ }^{184}$, V. Nikolaenko ${ }^{170, a j}$, I. Nikolic-Audit ${ }^{111}$, K. Nikolopoulos ${ }^{21}$, J. K. Nilsen ${ }^{151}$, P. Nilsson ${ }^{36}$, Y. Ninomiya ${ }^{206}$, A. Nisati ${ }^{172}$, N. Nishu ${ }^{52}$, R. Nisius ${ }^{132}$, T. Nobe ${ }^{206}$, Y. Noguchi ${ }^{98}$, M. Nomachi ${ }^{150}$, I. Nomidis ${ }^{45}$, M. A. Nomura ${ }^{36}$, T. Nooney ${ }^{107}$, M. Nordberg ${ }^{46}$, N. Norjoharuddeen ${ }^{152}$, O. Novgorodova ${ }^{68}$, S. Nowak ${ }^{132}$, M. Nozaki $^{96}$, L. Nozka ${ }^{147}$, K. Ntekas ${ }^{217}$, E. Nurse ${ }^{109}$, F. Nuti ${ }^{119}$, D. C. O'Neil ${ }^{189}$, A. A. O'Rourke ${ }^{66}$, V. O'Shea ${ }^{79}$, F. G. Oakham ${ }^{45, d}$, H. Oberlack ${ }^{132}$, T. Obermann ${ }^{29}$, J. Ocariz ${ }^{111}$, A. Ochi ${ }^{17}$, I. Ochoa ${ }^{57}$, J. P. Ochoa-Ricoux ${ }^{48}$, S. Oda ${ }^{100}$, S. Odaka ${ }^{96}$, H. Ogren ${ }^{91}$, A. Oh ${ }^{115}$, S. H. Oh ${ }^{69}$, C. C. Ohm ${ }^{18}$, H. Ohman ${ }^{221}$, H. Oide ${ }^{74,75}$, H. Okawa ${ }^{215}$, Y. Okumura ${ }^{206}$, T. Okuyama ${ }^{96}$, A. Olariu ${ }^{38}$, L. F. Oleiro Seabra ${ }^{160}$, S. A. Olivares Pino ${ }^{70}$, D. Oliveira Damazio ${ }^{36}$, A. Olszewski ${ }^{63}$, J. Olszowska ${ }^{63}$, A. Onofre ${ }^{160,164}$, K. Onogi ${ }^{134}$, P. U. E. Onyisi ${ }^{13,2}$, M. J. Oreglia ${ }^{47}$, Y. Oren ${ }^{204}$, D. Orestano ${ }^{176,177}$ N. Orlando ${ }^{88}$, R. S. Orr ${ }^{210}$, B. Osculati ${ }^{74,75}$, R. Ospanov ${ }^{115}$, G. Otero y Garzon ${ }^{43}$, H. Otono ${ }^{100}$, M. Ouchrif ${ }^{181}$, F. Ould-Saada ${ }^{151}$, A. Ouraou ${ }^{183}$, K. P. Oussoren ${ }^{139}$, Q. Ouyang ${ }^{50}$, M. Owen ${ }^{79}$, R. E. Owen ${ }^{21}$, V. E. Ozcan ${ }^{22}$, N. Ozturk ${ }^{10}$, K. Pachal ${ }^{189}$, A. Pacheco Pages ${ }^{15}$, L. Pacheco Rodriguez ${ }^{183}$, C. Padilla Aranda ${ }^{15}$, S. Pagan Griso ${ }^{18}$, M. Paganini ${ }^{232}$, F. Paige ${ }^{36}$, P. Pais ${ }^{117}$, G. Palacino ${ }^{91}$, S. Palazzo 59,60, S. Palestini ${ }^{46}$, M. Palka ${ }^{62}$, D. Pallin ${ }^{56}$, E. St. Panagiotopoulou ${ }^{12}$, I. Panagoulias ${ }^{12}$, C. E. Pandini ${ }^{111}$, J. G. Panduro Vazquez ${ }^{108}$, P. Pani ${ }^{46}$, S. Panitkin ${ }^{36}$, D. Pantea ${ }^{38}$, L. Paolozzi ${ }^{73}$, Th. D. Papadopoulou'2, K. Papageorgiou ${ }^{11}$, A. Paramonov ${ }^{8}$, D. Paredes Hernandez ${ }^{232}$, A. J. Parker ${ }^{102}$, M. A. Parker ${ }^{44}$, K. A. Parker ${ }^{66}$, F. Parodi $^{74,75}$, J. A. Parsons ${ }^{57}$, U. Parzefall 72 , V. R. Pascuzzi ${ }^{210}$, J. M. Pasner ${ }^{184}$, E. Pasqualucci ${ }^{172}$, S. Passaggio ${ }^{74}$, Fr. Pastore ${ }^{108}$, S. Pataraia ${ }^{231}$, J. R. Pater ${ }^{115}$, T. Pauly ${ }^{46}$, J. Pearce ${ }^{225}$, B. Pearson ${ }^{132}$, L. E. Pedersen ${ }^{58}$, S. Pedraza Lopez ${ }^{223}$, R. Pedro ${ }^{160,161}$, S. V. Peleganchuk ${ }^{141, c}$, O. Penc $^{167}$, C. Peng ${ }^{50}$, H. Peng ${ }^{53}$, J. Penwell ${ }^{91}$, B. S. Peralva ${ }^{33}$, M. M. Perego ${ }^{183}$, D. V. Perepelitsa ${ }^{36}$, L. Perini ${ }^{122,123}$, H. Pernegger $^{46}$, S. Perrella ${ }^{135,136}$, R. Peschke ${ }^{66}$, V. D. Peshekhonov ${ }^{95}$, K. Peters ${ }^{66}$, R. F. Y. Peters ${ }^{115}$, B. A. Petersen ${ }^{46}$, T. C. Petersen ${ }^{58}$, E. Petit ${ }^{81}$, A. Petridis ${ }^{1}$, C. Petridou 205 , P. Petroff ${ }^{149}$, E. Petrolo ${ }^{172}$, M. Petrov ${ }^{152}$, F. Petrucci ${ }^{176,177}$, N. E. Pettersson ${ }^{117}$, A. Peyaud $d^{183}$, R. Pezoa $^{49}$, P. W. Phillips ${ }^{171}$, G. Piacquadio ${ }^{199}$, E. Pianori ${ }^{226}$, A. Picazio ${ }^{117}$, E. Piccaro ${ }^{107}$, M. A. Pickering ${ }^{152}$, R. Piegaia ${ }^{43}$, J. E. Pilcher ${ }^{47}$, A. D. Pilkington ${ }^{115}$, A. W. J. Pin ${ }^{115}$, M. Pinamonti $218,220, a l$, J. L. Pinfold ${ }^{3}$, H. Pirumov ${ }^{66}$, M. Pitt ${ }^{228}$, L. Plazak ${ }^{191}$, M.-A. Pleier ${ }^{36}$, V. Pleskot ${ }^{114}$, E. Plotnikova ${ }^{95}$, D. Pluth ${ }^{94}$, P. Podberezko ${ }^{141}$, R. Poettgen ${ }^{196,197}$, L. Poggioli ${ }^{149}$, D. Pohl29 ${ }^{29}$, G. Polesello ${ }^{153}$, A. Poley ${ }^{66}$, A. Policicchio ${ }^{59,60}$, R. Polifka ${ }^{46}$, A. Polini ${ }^{27}$, C. S. Pollard ${ }^{79}$, V. Polychronakos ${ }^{36}$, K. Pommès ${ }^{46}$, D. Ponomarenko ${ }^{129}$, L. Pontecorvo ${ }^{172}$, B. G. Pope ${ }^{121}$, G. A. Popeneciu ${ }^{40}$, A. Poppleton ${ }^{46}$, S. Pospisil ${ }^{168}$, K. Potamianos ${ }^{18}$, I. N. Potrap ${ }^{95}$, C. J. Potter ${ }^{44}$, C. T. Potter ${ }^{148}$, G. Poulard ${ }^{46}$, J. Poveda ${ }^{46}$, M. E. Pozo Astigarraga ${ }^{46}$, P. Pralavorio ${ }^{116}$, A. Pranko ${ }^{18}$, S. Prell ${ }^{14}$, D. Price ${ }^{115}$, L. E. Price ${ }^{8}$, M. Primavera ${ }^{103}$, S. Prince ${ }^{118}$, N. Proklova ${ }^{129}$, K. Prokofiev ${ }^{89}$, F. Prokoshin ${ }^{49}$, S. Protopopescu ${ }^{36}$, J. Proudfoot ${ }^{8}$, M. Przybycien ${ }^{61}$, D. Puddu176,177, A. Puri222, P. Puzo ${ }^{149}$, J. Qian ${ }^{120}$, G. Qin ${ }^{79}$, Y. Qin ${ }^{115}$, A. Quadt ${ }^{80}$, W. B. Quayle 218,219 , M. Queitsch-Maitland ${ }^{66}$, D. Quilty ${ }^{79}$, S. Raddum ${ }^{151}$, V. Radeka ${ }^{36}$, V. Radescu ${ }^{152}$, S. K. Radhakrishnan ${ }^{199}$, P. Radloff ${ }^{148}$, P. Rados ${ }^{119}$, F. Ragusa ${ }^{122,123}$, G. Rahal ${ }^{235}$, J. A. Raine ${ }^{115}$, S. Rajagopalan ${ }^{36}$, C. Rangel-Smith ${ }^{221}$, M. G. Ratti ${ }^{122,123}$, D. M. Rauch ${ }^{66}$, F. Rauscher ${ }^{131}$, S. Rave ${ }^{114}$, T. Ravenscroft ${ }^{79}$, I. Ravinovich ${ }^{228}$, J. H. Rawling ${ }^{115}$, M. Raymond ${ }^{46}$, A. L. Read ${ }^{151}$, N. P. Readioff ${ }^{105}$, M. Reale ${ }^{103,104}$, D. M. Rebuzzi ${ }^{153,154}$, A. Redelbach"230, G. Redlinger ${ }^{36}$, R. Reece ${ }^{184}$, R. G. Reed ${ }^{195}$, K. Reeves $^{65}$, L. Rehnisch ${ }^{19}$, J. Reichert ${ }^{155}$, A. Reiss ${ }^{114}$, C. Rembser ${ }^{46}$, H. Ren ${ }^{50}$, M. Rescigno ${ }^{172}$, S. Resconi1 ${ }^{122}$, E. D. Resseguie ${ }^{155}$, S. Rettie ${ }^{224}$, E. Reynolds ${ }^{21}$, O. L. Rezanova1 ${ }^{141, c}$, P. Reznicek ${ }^{169}$, R. Rezvani ${ }^{126}$, R. Richter ${ }^{132}$, S. Richter ${ }^{109}$, E. Richter-Was ${ }^{62}$, O. Ricken ${ }^{29}$, M. Ridel ${ }^{111}$, P. Rieck ${ }^{132}$, C. J. Riegel ${ }^{231}$, J. Rieger ${ }^{80}$, O. Rifki ${ }^{145}$, M. Rijssenbeek ${ }^{199}$, A. Rimoldi153,154, M. Rimoldi ${ }^{20}$, L. Rinaldi 27 , B. Ristić ${ }^{73}$, E. Ritsch $^{46}$, I. Riu ${ }^{15}$, F. Rizatdinova ${ }^{146}$, E. Rizvi107, C. Rizzi15, R. T. Roberts'115, S. H. Robertson 118,0, A. Robichaud-Veronneau ${ }^{118}$, D. Robinson $^{44}$, J. E. M. Robinson ${ }^{66}$, A. Robson ${ }^{79}$, C. Roda ${ }^{157,158}$, Y. Rodina ${ }^{116, a m}$, A. Rodriguez Perez ${ }^{15}$, D. Rodriguez Rodriguez ${ }^{223}$, S. Roe ${ }^{46}$, C. S. Rogan ${ }^{82}$, O. Røhne ${ }^{151}$, J. Roloff ${ }^{22}$, A. Romaniouk'129, M. Romano ${ }^{27,28}$, S. M. Romano Saez ${ }^{56}$, E. Romero Adam ${ }^{223}$, N. Rompotis ${ }^{105}$, M. Ronzani ${ }^{72}$, L. Roos ${ }^{111}$, S. Rosati ${ }^{172}$, K. Rosbach'72, P. Rose ${ }^{184}$, N.-A. Rosien ${ }^{80}$, V. Rossetti ${ }^{196,197}$, E. Rossi ${ }^{135,136}$, L. P. Rossi ${ }^{74}$, J. H. N. Rosten ${ }^{44}$, R. Rosten ${ }^{185}$, M. Rotaru ${ }^{38}$, I. Roth ${ }^{228}$, J. Rothberg ${ }^{185}$, D. Rousseau ${ }^{149}$, A. Rozanov ${ }^{116}$, Y. Rozen ${ }^{203}$, X. Ruan ${ }^{195}$, F. Rubbo ${ }^{190}$, F. Rühr ${ }^{72}$, A. Ruiz-Martinez ${ }^{45}$, Z. Rurikova ${ }^{72}$, N. A. Rusakovich ${ }^{95}$, A. Ruschke ${ }^{131}$, H. L. Russell ${ }^{185}$, J. P. Rutherfoord9 , N. Ruthmann ${ }^{46}$, Y. F. Ryabov' ${ }^{156}$, M. Rybar ${ }^{222}$, G. Rybkin ${ }^{149}$, S. Ryu ${ }^{8}$, A. Ryzhov ${ }^{170}$, G. F. Rzehorz ${ }^{80}$, A. F. Saavedra ${ }^{201}$, G. Sabato ${ }^{139}$, S. Sacerdoti ${ }^{43}$, H. F.-W. Sadrozinski ${ }^{184}$, R. Sadykov ${ }^{95}$, F. Safai Tehrani ${ }^{172}$, P. Saha ${ }^{140}$, M. Sahinsoy ${ }^{83}$, M. Saimpert ${ }^{66}$, M. Saito ${ }^{206}$, T. Saito ${ }^{206}$, H. Sakamoto ${ }^{206}$, Y. Sakurai ${ }^{227}$, G. Salamanna ${ }^{176,177}$, J. E. Salazar Loyola ${ }^{49}$, D. Salek 139 , P. H. Sales De Bruin ${ }^{221}$, D. Salihagic ${ }^{132}$, A. Salnikov ${ }^{190}$, J. Salt ${ }^{223}$, D. Salvatore ${ }^{59,60,}$ F. Salvatore ${ }^{200}$, A. Salvucci ${ }^{87,88,89}$, A. Salzburger ${ }^{46}$, D. Sammel ${ }^{72}$, D. Sampsonidis ${ }^{205}$, J. Sánchez ${ }^{223}$, V. Sanchez Martinez ${ }^{223}$, A. Sanchez Pineda ${ }^{218,220}$, H. Sandaker ${ }^{151}$, R. L. Sandbach ${ }^{107}$, C. O. Sander ${ }^{66}$, M. Sandhoff ${ }^{231}$, C. Sandoval26, D. P. C. Sankey ${ }^{171}$, M. Sannino ${ }^{74,75}$, A. Sansoni ${ }^{71}$, C. Santoni ${ }^{56}$, R. Santonico ${ }^{174,175}$, H. Santos ${ }^{160}$, I. Santoyo Castillo ${ }^{200}$, K. Sapp ${ }^{159}$, A. Sapronov ${ }^{95}$, J. G. Saraiva ${ }^{160,163}$, B. Sarrazin ${ }^{29}$, O. Sasaki ${ }^{96}$, K. Sato ${ }^{215}$, E. Sauvan77, G. Savage ${ }^{108}$, P. Savard²10, , N. Savic ${ }^{132}$, C. Sawyer ${ }^{171}$, L. Sawyer ${ }^{110,4}$, J. Saxon ${ }^{47}$, C. Sbarra ${ }^{27}$, A. Sbrizzi ${ }^{27,28}$, T. Scanlon ${ }^{109}$, D. A. Scannicchio 217 , M. Scarcella 201 , V. Scarfone ${ }^{59,60,}$ J. Schaarschmidt ${ }^{185}$, P. Schacht ${ }^{132}$, B. M. Schachtner ${ }^{131}$, D. Schaefer ${ }^{46}$, L. Schaefer ${ }^{155}$, R. Schaefer ${ }^{66}$, J. Schaeffer ${ }^{114}$, S. Schaepe ${ }^{29}$, S. Schaetzel ${ }^{84}$, U. Schäfer ${ }^{114}$, A. C. Schaffer ${ }^{149}$, D. Schaile ${ }^{131}$, R. D. Schamberger ${ }^{199}$, V. Scharf ${ }^{83}$, V. A. Schegelsky ${ }^{156}$, D. Scheirich ${ }^{169}$, M. Schernau ${ }^{217}$, C. Schiavi ${ }^{74,75}$, S. Schier ${ }^{184}$, L. K. Schildgen ${ }^{29}$, C. Schillo ${ }^{72}$, M. Schioppa ${ }^{59,60}$, S. Schlenker ${ }^{46}$, K. R. Schmidt-Sommerfeld ${ }^{132}$, K. Schmieden ${ }^{46}$, C. Schmitt ${ }^{114}$, S. Schmitt ${ }^{66}$, S. Schmitz ${ }^{114}$, U. Schnoor ${ }^{72}$, L. Schoeffel ${ }^{183}$, A. Schoening ${ }^{84}$, B. D. Schoenrock ${ }^{121}$, E. Schopf ${ }^{29}$, M. Schott ${ }^{114}$, J. F. P. Schouwenberg ${ }^{138}$, J. Schovancova ${ }^{234}$, S. Schramm ${ }^{73}$, N. Schuh ${ }^{114}$, A. Schulte ${ }^{114}$, M. J. Schultens ${ }^{29}$, H.-C. Schultz-Coulon ${ }^{83}$, H. Schulz ${ }^{19}$, M. Schumacher ${ }^{72}$, B. A. Schumm ${ }^{184}$, Ph. Schune ${ }^{183}$, A. Schwartzman ${ }^{190}$, T. A. Schwarz ${ }^{120}$, H. Schweiger ${ }^{115}$, Ph. Schwemling ${ }^{183}$, R. Schwienhorst ${ }^{121}$, J. Schwindling ${ }^{183}$, T. Schwindt ${ }^{29}$, A. Sciandra ${ }^{29}$, G. Sciolla ${ }^{31}$, F. Scuri ${ }^{157,158}$, F. Scutti ${ }^{119}$, J. Searcy ${ }^{120}$, P. Seema ${ }^{29}$, S. C. Seidel ${ }^{137}$, 
A. Seiden ${ }^{184}$, J. M. Seixas ${ }^{32}$, G. Sekhniaidze ${ }^{135}$, K. Sekhonn ${ }^{120}$, S. J. Sekula ${ }^{64}$, N. Semprini-Cesari27,28, C. Serfon ${ }^{151}$, L. Serin ${ }^{149}$, L. Serkin ${ }^{218,219}$, M. Sessa ${ }^{176,177}$, R. Seuster ${ }^{225}$, H. Severini ${ }^{145}$, T. Sfiligoj ${ }^{106}$, F. Sforza ${ }^{46}$, A. Sfyrla ${ }^{73}$, E. Shabalina ${ }^{80}$,

N. W. Shaikh ${ }^{196,197}$, L. Y. Shan ${ }^{50}$, R. Shang ${ }^{222}$, J. T. Shank ${ }^{30}$, M. Shapiro ${ }^{18}$, P. B. Shatalov ${ }^{128}$, K. Shaw ${ }^{218,219}$, S. M. Shaw ${ }^{115}$, A. Shcherbakova ${ }^{196,197}$, C. Y. Shehu ${ }^{200}$, Y. Shen ${ }^{145}$, P. Sherwood ${ }^{109}$, L. Shi ${ }^{202, a n}$, S. Shimizu ${ }^{97}$, C. O. Shimmin ${ }^{232}$, M. Shimojima ${ }^{133}$, S. Shirabe ${ }^{100}$, M. Shiyakova ${ }^{95, a 0}$, J. Shlomi $i^{228}$, A. Shmeleva ${ }^{127}$, D. Shoaleh Saadi' ${ }^{126}$, M. J. Shochet ${ }^{47}$, S. Shojaii' ${ }^{122}$, D. R. Shope ${ }^{145}$, S. Shrestha ${ }^{143}$, E. Shulga ${ }^{129}$, M. A. Shupe ${ }^{9}$, P. Sicho ${ }^{167}$, A. M. Sickles ${ }^{222}$, P. E. Sidebo ${ }^{198}$, E. Sideras Haddad ${ }^{195}$, O. Sidiropoulou ${ }^{230}$, D. Sidorov ${ }^{146}$, A. Sidoti2 ${ }^{27,28}$, F. Siegert ${ }^{68}$, Dj. Sijacki ${ }^{16}$, J. Silva ${ }^{160,163}$, S. B. Silverstein ${ }^{196}$, V. Simak ${ }^{168}$, Lj. Simic ${ }^{16}$, S. Simion ${ }^{149}$, E. Simioni ${ }^{114}$, B. Simmons ${ }^{109}$, M. Simon ${ }^{114}$, P. Sinervo ${ }^{210}$, N. B. Sinev ${ }^{148}$, M. Sioli27,28, G. Siragusa ${ }^{230}$, I. Siral ${ }^{120}$,

S. Yu. Sivoklokov ${ }^{130}$, J. Sjölin ${ }^{196,197}$, M. B. Skinner ${ }^{102}$, P. Skubic ${ }^{145}$, M. Slater ${ }^{21}$, T. Slavicek ${ }^{168}$, M. Slawinska ${ }^{139}$, K. Sliwa ${ }^{216}$, R. Slovak ${ }^{169}$, V. Smakhtin 228 , B. H. Smart ${ }^{7}$, J. Smiesko ${ }^{191}$, N. Smirnov ${ }^{129}$, S. Yu. Smirnov ${ }^{129}$, Y. Smirnov ${ }^{129}$, L. N. Smirnova ${ }^{130, a p, ~}$ O. Smirnova912, J. W. Smith ${ }^{80}$, M. N. K. Smith ${ }^{57}$, R. W. Smith ${ }^{57}$, M. Smizanska ${ }^{102}$, K. Smolek ${ }^{168}$, A. A. Snesarev ${ }^{127}$, I. M. Snyder ${ }^{148}$, S. Snyder ${ }^{36}$, R. Sobie ${ }^{225,0}$, F. Socher ${ }^{68}$, A. Soffer ${ }^{204}$, D. A. Soh ${ }^{202}$, G. Sokhrannyi ${ }^{106}$, C. A. Solans Sanchez ${ }^{46}$, M. Solar ${ }^{168}$, E. Yu. Soldatov ${ }^{129}$, U. Soldevila223, A. A. Solodkov ${ }^{170}$, A. Soloshenko ${ }^{95}$, O. V. Solovyanov ${ }^{170}$, V. Solovyev ${ }^{156}$, P. Sommer ${ }^{72}$, H. Son ${ }^{216}$, H. Y. Song $53, a 9$, A. Sopczak ${ }^{168}$, V. Sorin ${ }^{15}$, D. Sosa ${ }^{84}$, C. L. Sotiropoulou ${ }^{157,158}$, R. Soualah ${ }^{218,220}$, A. M. Soukharev ${ }^{141, c}$, D. South ${ }^{66}$, B. C. Sowden ${ }^{108}$, S. Spagnolo ${ }^{103,104}$, M. Spalla ${ }^{157,158}$, M. Spangenberg 226 , F. Spanò ${ }^{108}$, D. Sperlich ${ }^{19}$, F. Spettel ${ }^{132}$, T. M. Spieker ${ }^{83}$, R. Spighi ${ }^{27}$, G. Spigo ${ }^{46}$, L. A. Spiller ${ }^{119}$, M. Spousta ${ }^{169}$, R. D. St. Denis ${ }^{79}$, A. Stabile ${ }^{122}$, R. Stamen ${ }^{83}$, S. Stamm ${ }^{19}$, E. Stanecka ${ }^{63}$, R. W. Stanek ${ }^{8}$, C. Stanescu' ${ }^{176}$, M. M. Stanitzki ${ }^{66}$, S. Stapnes ${ }^{151}$, E. A. Starchenko ${ }^{170,}$ G. H. Stark ${ }^{47}$, J. Stark ${ }^{81}$, S. H. Stark ${ }^{58}$, P. Staroba ${ }^{167}$, P. Starovoitov ${ }^{83}$, S. Stärz ${ }^{46}$, R. Staszewski ${ }^{63}$, P. Steinberg ${ }^{36}$, B. Stelzer ${ }^{189}$, H. J. Stelzer ${ }^{46}$, O. Stelzer-Chilton ${ }^{213}$, H. Stenzel ${ }^{78}$, G. A. Stewart ${ }^{79}$, J. A. Stillings ${ }^{29}$, M. C. Stockton ${ }^{148}$, M. Stoebe ${ }^{118}$, G. Stoicea ${ }^{38}$, P. Stolte ${ }^{80}$, S. Stonjek ${ }^{132}$, A. R. Stradling ${ }^{10}$, A. Straessner ${ }^{68}$, M. E. Stramaglia ${ }^{20}$, J. Strandberg ${ }^{198}$, S. Strandberg ${ }^{196,197}$, A. Strandlie ${ }^{151}$, M. Strauss ${ }^{145}$, P. Strizenec ${ }^{192}$, R. Ströhmer ${ }^{230}$, D. M. Strom ${ }^{148}$, R. Stroynowski ${ }^{64}$ A. Strubig ${ }^{138}$, S. A. Stucci ${ }^{36}$, B. Stugu' ${ }^{17}$, N. A. Styles ${ }^{66}$, D. Su ${ }^{190}$, J. Su ${ }^{159}$, S. Suchek ${ }^{83}$, Y. Sugaya ${ }^{150}$, M. Suk ${ }^{168}$, V. V. Sulin ${ }^{127}$, S. Sultansoy ${ }^{6}$, T. Sumida ${ }^{98}$, S. Sun ${ }^{82}$, X. Sun ${ }^{3}$, K. Suruliz ${ }^{200}$, C. J. E. Suster ${ }^{201}$, M. R. Sutton ${ }^{200}$, S. Suzuki $i^{96}$, M. Svatos ${ }^{167}$, M. Swiatlowski ${ }^{47}$, S. P. Swift², A. Sydorenko ${ }^{114}$, I. Sykora ${ }^{191}$, T. Sykora ${ }^{169}$, D. Ta ${ }^{72}$, K. Tackmann ${ }^{66}$, J. Taenzer ${ }^{204}$, A. Taffard ${ }^{217}$, R. Tafirout ${ }^{213}$, N. Taiblum ${ }^{204}$, H. Takai ${ }^{36}$, R. Takashima ${ }^{99}$, T. Takeshita ${ }^{187}$, Y. Takubo ${ }^{96}$, M. Talby ${ }^{116}$, A. A. Talyshev ${ }^{141, c}$, J. Tanaka206, M. Tanaka208, R. Tanaka ${ }^{149}$, S. Tanaka ${ }^{96}$, R. Tanioka ${ }^{97}$, B. B. Tannenwald ${ }^{143}$, S. Tapia Araya ${ }^{49}$, S. Tapprogge ${ }^{114}$, S. Tarem ${ }^{203}$, G. F. Tartarelli ${ }^{122}$, P. Tas ${ }^{169}$, M. Tasevsky ${ }^{167}$, T. Tashiro ${ }^{98}$, E. Tassi ${ }^{59,60}$, A. Tavares Delgado 160,161 , Y. Tayalati ${ }^{182}$, A. C. Taylor ${ }^{137}$, G. N. Taylor ${ }^{119}$, P. T. E. Taylor ${ }^{119}$, W. Taylor ${ }^{214}$, P. Teixeira-Dias ${ }^{108}$, D. Temple ${ }^{189}$, H. Ten Kate ${ }^{46}$, P. K. Teng ${ }^{202}$, J. J. Teoh ${ }^{150}$, F. Tepe ${ }^{231}$, S. Terada ${ }^{96}$, K. Terashi ${ }^{206}$, J. Terron ${ }^{113}$, S. Terzo ${ }^{15}$, M. Testa ${ }^{71}$, R. J. Teuscher ${ }^{210,0}$, T. Theveneaux-Pelzer ${ }^{116}$, J. P. Thomas ${ }^{21}$, J. Thomas-Wilsker ${ }^{108}$, P. D. Thompson ${ }^{21}$, A. S. Thompson ${ }^{79}$, L. A. Thomsen ${ }^{232}$, E. Thomson ${ }^{155}$, M. J. Tibbetts ${ }^{18,}$ R. E. Ticse Torres ${ }^{116}$, V. O. Tikhomirov ${ }^{127, a r}$, Yu. A. Tikhonov ${ }^{141, c}$, S. Timoshenko ${ }^{129}$, P. Tipton ${ }^{232}$, S. Tisserant ${ }^{116}$, K. Todome ${ }^{208}$, S. Todorova-Nova7, J. Tojo ${ }^{100}$, S. Tokár ${ }^{191}$, K. Tokushuku' ${ }^{96}$, E. Tolley ${ }^{82}$, L. Tomlinson ${ }^{115}$, M. Tomoto ${ }^{134}$, L. Tompkins ${ }^{190, a s,}$ K. Toms ${ }^{137}$, B. Tong ${ }^{82}$, P. Tornambe ${ }^{72}$, E. Torrence ${ }^{148}$, H. Torres ${ }^{189}$, E. Torró Pastor ${ }^{185}$, J. Toth ${ }^{116, a t}$, F. Touchard ${ }^{116}$, D. R. Tovey ${ }^{186}$, C. J. Treado ${ }^{142}$, T. Trefzger ${ }^{230}$, A. Tricoli ${ }^{36}$, I. M. Trigger ${ }^{213}$, S. Trincaz-Duvoidd ${ }^{111}$, M. F. Tripiana ${ }^{15}$, W. Trischuk ${ }^{210}$, B. Trocmé81,

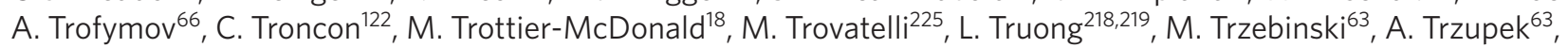
K. W. Tsang87, J. C.-L. Tseng152, P. V. Tsiareshka ${ }^{124}$, G. Tsipolitis ${ }^{12}$, N. Tsirintanis 11 , S. Tsiskaridze ${ }^{15}$, V. Tsiskaridze ${ }^{72}$,

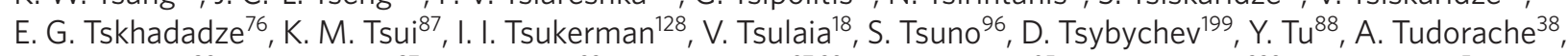
V. Tudorache ${ }^{38}$, T. T. Tulbure ${ }^{37}$, A. N. Tuna ${ }^{82}$, S. A. Tupputi2 ${ }^{27,28}$, S. Turchikhin ${ }^{95}$, D. Turgeman ${ }^{228}$, I. Turk Cakir 5,au, R. Turra ${ }^{122,123,}$

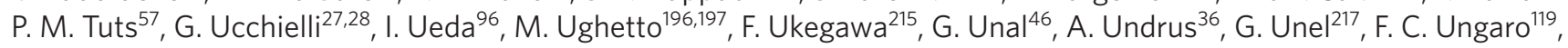
Y. Unno ${ }^{96}$, C. Unverdorben ${ }^{131}$, J. Urban ${ }^{192}$, P. Urquijo ${ }^{119}$, P. Urrejola ${ }^{114}$, G. Usai ${ }^{10}$, J. Usui ${ }^{96}$, L. Vacavant ${ }^{116}$, V. Vacek ${ }^{168}$, B. Vachon ${ }^{118}$, C. Valderanis ${ }^{131}$, E. Valdes Santurio ${ }^{196,197}$, N. Valencic ${ }^{139}$, S. Valentinetti2 ${ }^{27,28}$, A. Valero ${ }^{223}$, L. Valéry ${ }^{15}$, S. Valkar ${ }^{169}$, A. Vallier ${ }^{7}$, J. A. Valls Ferrer ${ }^{223}$, W. Van Den Wollenberg ${ }^{139}$, H. van der Graaf ${ }^{139}$, N. van Eldik ${ }^{203}$, P. van Gemmeren ${ }^{8}$,

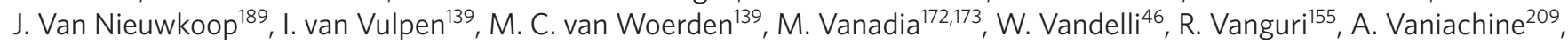
P. Vankov ${ }^{139}$, G. Vardanyan ${ }^{233}$, R. Vari ${ }^{172}$, E. W. Varnes ${ }^{9}$, C. Varni ${ }^{74,75}$, T. Varol ${ }^{64}$, D. Varouchas ${ }^{149}$, A. Vartapetian ${ }^{10}$, K. E. Varvell ${ }^{201}$, J. G. Vasquez 232 , G. A. Vasquez ${ }^{49}$, F. Vazeille ${ }^{56}$, T. Vazquez Schroeder ${ }^{118}$, J. Veatch ${ }^{80}$, V. Veeraraghavan ${ }^{9}$, L. M. Veloce ${ }^{210}$, F. Veloso ${ }^{160,162}$, S. Veneziano ${ }^{172}$, A. Ventura ${ }^{103,104}$, M. Venturi ${ }^{225}$, N. Venturi210, A. Venturini ${ }^{31}$, V. Vercesi ${ }^{153}$, M. Verducci ${ }^{176,177}$, W. Verkerke ${ }^{139}$, J. C. Vermeulen ${ }^{139}$, M. C. Vetterli" ${ }^{189, d}$, N. Viaux Maira ${ }^{49}$, O. Viazlo ${ }^{112}$, I. Vichou ${ }^{222}$, T. Vickey ${ }^{186}$, O. E. Vickey Boeriu'186, G. H. A. Viehhauser ${ }^{152}$, S. Viel ${ }^{18}$, L. Vigani ${ }^{152}$, M. Villa ${ }^{27,28}$, M. Villaplana Perez ${ }^{122,123}$ E. Vilucchi ${ }^{71}$, M. G. Vincter ${ }^{45}$, V. B. Vinogradov ${ }^{95}$, A. Vishwakarma ${ }^{66}$, C. Vittori ${ }^{27,28}$, I. Vivarelli200, S. Vlachos ${ }^{12}$, M. Vlasak ${ }^{168}$, M. Voge ${ }^{231}$, P. Vokac ${ }^{168}$, G. Volpi ${ }^{157,158}$, M. Volpi ${ }^{199}$, H. von der Schmitt ${ }^{132}$, E. von Toerne ${ }^{29}$, V. Vorobel ${ }^{169}$, K. Vorobev $^{129}$, M. Vos ${ }^{223}$, R. Voss ${ }^{46}$, J. H. Vossebeld ${ }^{105}$, N. Vranjes ${ }^{16}$, M. Vranjes Milosavljevic ${ }^{16}, V^{2}$. Vrba ${ }^{168}$, M. Vreeswijk ${ }^{139}$, R. Vuillermet ${ }^{46}$, I. Vukotic ${ }^{47}$, P. Wagner ${ }^{29}$, W. Wagner ${ }^{231}$, H. Wahlberg ${ }^{101}$, S. Wahrmund ${ }^{68}$, J. Wakabayashi' ${ }^{134}$, J. Walder ${ }^{102}$, R. Walker ${ }^{131}$, W. Walkowiak ${ }^{188}$, V. Wallangen ${ }^{196,197}$, C. Wang ${ }^{51}$, C. Wang ${ }^{54, a v}$, F. Wang ${ }^{229}$, H. Wang ${ }^{18}$, H. Wang ${ }^{3}$, J. Wang 66 , J. Wang ${ }^{201}$, Q. Wang ${ }^{145}$, R. Wang 8 , S. M. Wang ${ }^{202}$, T. Wang ${ }^{57}$, W. Wang ${ }^{202, a w}$, W. Wang ${ }^{53}$, Z. Wang ${ }^{55}$, C. Wanotayaroj ${ }^{148}$, A. Warburton ${ }^{118}$, C. P. Ward ${ }^{44}$, D. R. Wardrope ${ }^{109}$, A. Washbrook ${ }^{70}$, P. M. Watkins ${ }^{21}$, A. T. Watson ${ }^{21}$, M. F. Watson ${ }^{21}$, G. Watts ${ }^{185}$, S. Watts ${ }^{115}$, B. M. Waugh ${ }^{109}$, A. F. Webb ${ }^{13}$, S. Webb ${ }^{114}$, M. S. Weber 20, S. W. Weber ${ }^{230}$, S. A. Weber ${ }^{45}$, J. S. Webster ${ }^{8}$, A. R. Weidberg ${ }^{152}$, B. Weinert ${ }^{91}$, J. Weingarten ${ }^{80}$, C. Weiser ${ }^{72}$, H. Weits ${ }^{139}$, P. S. Wells ${ }^{46}$, T. Wenaus $^{36}$, T. Wengler ${ }^{46}$, S. Wenig ${ }^{46}$, N. Wermes ${ }^{29}$, 
M. D. Werner ${ }^{94}$, P. Werner ${ }^{46}$, M. Wessels ${ }^{83}$, K. Whalen $^{148}$, N. L. Whallon ${ }^{185}$, A. M. Wharton ${ }^{102}$, A. White ${ }^{10}$, M. J. White ${ }^{1}$, R. White ${ }^{49}$, D. Whiteson ${ }^{217}$, F. J. Wickens ${ }^{171}$, W. Wiedenmann ${ }^{229}$, M. Wielers ${ }^{171}$, C. Wiglesworth ${ }^{58}$, L. A. M. Wiik-Fuchs ${ }^{29}$, A. Wildauer ${ }^{132}$, F. Wilk ${ }^{115}$, H. G. Wilkens ${ }^{46}$, H. H. Williams ${ }^{155}$, S. Williams ${ }^{139}$, C. Willis ${ }^{121}$, S. Willocq ${ }^{117}$, J. A. Wilson ${ }^{21}$, I. Wingerter-Seez ${ }^{7}$, F. Winklmeier ${ }^{148}$, O. J. Winston ${ }^{200}$, B. T. Winter ${ }^{29}$, M. Wittgen ${ }^{190}$, M. Wobisch ${ }^{110,4}$, T. M. H. Wolf ${ }^{139}$, R. Wolff ${ }^{116}$, M. W. Wolter ${ }^{63}$, H. Wolters ${ }^{160,162}$, S. D. Worm²1, B. K. Wosiek ${ }^{63}$, J. Wotschack ${ }^{46}$, M. J. Woudstra ${ }^{115}$, K. W. Wozniak ${ }^{63}$, M. Wu ${ }^{47}$, S. L. Wu ${ }^{229}$, X. Wu ${ }^{73}$, Y. Wu ${ }^{120}$, T. R. Wyatt ${ }^{115}$, B. M. Wynne ${ }^{70}$, S. Xella ${ }^{58}$, Z. Xi ${ }^{120}$, L. Xia ${ }^{52}$, D. Xu $u^{50}$, L. Xu ${ }^{36}$, B. Yabsley 201 , S. Yacoob ${ }^{193}$, D. Yamaguchi ${ }^{208}$, Y. Yamaguchi ${ }^{150}$, A. Yamamoto ${ }^{96}$, S. Yamamoto ${ }^{206}$, T. Yamanakaa6, K. Yamauchi ${ }^{134}$, Y. Yamazaki ${ }^{97}$, Z. Yan ${ }^{30}$, H. Yang ${ }^{55}$, H. Yang ${ }^{18}$, Y. Yang ${ }^{202}$, Z. Yang ${ }^{17}$, W.-M. Yao ${ }^{18}$, Y. C. Yap ${ }^{111}$, Y. Yasu ${ }^{96}$, E. Yatsenko ${ }^{7}$, K. H. Yau Wong ${ }^{29}$, J. Ye ${ }^{64}$, S. Ye ${ }^{36}$, I. Yeletskikh ${ }^{95}$, E. Yigitbasi ${ }^{30}$, E. Yildirim ${ }^{114}$, K. Yorita ${ }^{227}$, K. Yoshihara ${ }^{155}$, C. Young ${ }^{190}$, C. J. S. Young ${ }^{46}$, S. Youssef ${ }^{30}$, D. R. Yu ${ }^{18}$, J. Yu ${ }^{10}$, J. Yu ${ }^{94}$, L. Yuan ${ }^{97}$, S. P. Y. Yuen ${ }^{29}$, I. Yusuff ${ }^{44, a x}$, B. Zabinski ${ }^{63}$ G. Zacharis ${ }^{12}$, R. Zaidan ${ }^{15}$, A. M. Zaitsev ${ }^{170, a j}$, N. Zakharchuk ${ }^{66}$, J. Zalieckas ${ }^{17}$, A. Zamann ${ }^{199}$, S. Zambito82, D. Zanzi119,

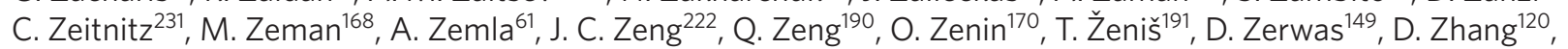

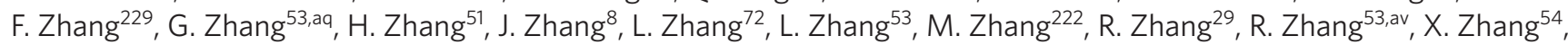
Y. Zhang ${ }^{50}$, Z. Zhang ${ }^{149}$, X. Zhao ${ }^{64}$, Y. Zhao ${ }^{54, a y}$, Z. Zhao ${ }^{53}$, A. Zhemchugov ${ }^{95}$, J. Zhong ${ }^{152}$, B. Zhou ${ }^{120}$, C. Zhou ${ }^{229}$, L. Zhou ${ }^{64}$,

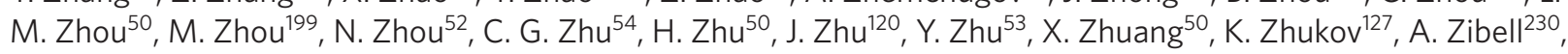
D. Zieminska ${ }^{91}$, N. I. Zimine ${ }^{95}$, C. Zimmermann ${ }^{114}$, S. Zimmermann ${ }^{72}$, Z. Zinonos ${ }^{132}$, M. Zinser ${ }^{114}$, M. Ziolkowski ${ }^{188}$, L. Živković ${ }^{16}$, G. Zobernig 229, A. Zoccoli²7,28, R. Zou ${ }^{47}$, M. zur Nedden ${ }^{19}$, L. Zwalinski ${ }^{46}$

\section{Primary affiliations}

${ }^{1}$ Department of Physics, University of Adelaide, Adelaide, Australia. ${ }^{2}$ Physics Department, SUNY Albany, Albany, New York, USA. ${ }^{3}$ Department of Physics, University of Alberta, Edmonton, Alberta, Canada. ${ }^{4}$ Department of Physics, Ankara University, Ankara, Turkey. ${ }^{5}$ Istanbul Aydin University, Istanbul, Turkey. ${ }^{6}$ Division of Physics, TOBB University of Economics and Technology, Ankara, Turkey. ${ }^{7}$ LAPP, CNRS/IN2P3 and Université Savoie Mont Blanc, Annecy-le-Vieux, France. ${ }^{8}$ High Energy Physics Division, Argonne National Laboratory, Argonne, Illinois, USA. ${ }^{9}$ Department of Physics, University of Arizona, Tucson, Arizona, USA. ${ }^{10}$ Department of Physics, The University of Texas at Arlington, Arlington, Texas, USA. ${ }^{11}$ Physics Department, National and Kapodistrian University of Athens, Athens, Greece. ${ }^{12}$ Physics Department, National Technical University of Athens, Zografou, Greece. ${ }^{13}$ Department of Physics, The University of Texas at Austin, Austin, Texas, USA. ${ }^{14}$ Institute of Physics, Azerbaijan Academy of Sciences, Baku, Azerbaijan. ${ }^{15}$ Institut de Física d'Altes Energies (IFAE), The Barcelona Institute of Science and Technology, Barcelona, Spain. ${ }^{16}$ Institute of Physics, University of Belgrade, Belgrade, Serbia. ${ }^{17}$ Department for Physics and Technology, University of Bergen, Bergen, Norway. ${ }^{18}$ Physics Division, Lawrence Berkeley National Laboratory and University of California, Berkeley, California, USA. ${ }^{19}$ Department of Physics, Humboldt University, Berlin, Germany. ${ }^{20}$ Albert Einstein Center for Fundamental Physics and Laboratory for High Energy Physics, University of Bern, Bern, Switzerland. ${ }^{21}$ School of Physics and Astronomy, University of Birmingham, Birmingham, UK. ${ }^{22}$ Department of Physics, Bogazici University, Istanbul, Turkey. ${ }^{23}$ Department of Physics Engineering, Gaziantep University, Gaziantep, Turkey. ${ }^{24}$ Istanbul Bilgi University, Faculty of Engineering and Natural Sciences, Istanbul, Turkey. ${ }^{25}$ Bahcesehir University, Faculty of Engineering and Natural Sciences, Istanbul, Turkey. ${ }^{26}$ Centro de Investigaciones, Universidad Antonio Narino, Bogota, Colombia. ${ }^{27}$ INFN Sezione di Bologna, Bologna, Italy. ${ }^{28}$ Dipartimento di Fisica e Astronomia, Università di Bologna, Bologna, Italy. ${ }^{29}$ Physikalisches Institut, University of Bonn, Bonn, Germany. ${ }^{30}$ Department of Physics, Boston University, Boston, Massachusetts, USA. ${ }^{31}$ Department of Physics, Brandeis University, Waltham, Massachusetts, USA. ${ }^{32}$ Universidade Federal do Rio De Janeiro COPPE/EE/IF, Rio de Janeiro, Brazil. ${ }^{33}$ Electrical Circuits Department, Federal University of Juiz de Fora (UFJF), Juiz de Fora, Brazil. ${ }^{34}$ Federal University of Sao Joao del Rei (UFSJ), Sao Joao del Rei, Brazil. ${ }^{35}$ Instituto de Fisica, Universidade de Sao Paulo, Sao Paulo, Brazil. ${ }^{36}$ Physics Department, Brookhaven National Laboratory, Upton, New York, USA. ${ }^{37}$ Transilvania University of Brasov, Brasov, Romania. ${ }^{38}$ Horia Hulubei National Institute of Physics and Nuclear Engineering, Bucharest, Romania. ${ }^{39}$ Department of Physics, Alexandru loan Cuza University of lasi, lasi, Romania.

${ }^{40}$ National Institute for Research and Development of Isotopic and Molecular Technologies, Physics Department, Cluj Napoca, Romania. ${ }^{41}$ University Politehnica Bucharest, Bucharest, Romania. ${ }^{42}$ West University in Timisoara, Timisoara, Romania. ${ }^{43}$ Departamento de Física, Universidad de Buenos Aires, Buenos Aires, Argentina. ${ }^{44}$ Cavendish Laboratory, University of Cambridge, Cambridge, UK. ${ }^{45}$ Department of Physics, Carleton University, Ottawa, Ontario, Canada. ${ }^{46}$ CERN, Geneva, Switzerland. ${ }^{47}$ Enrico Fermi Institute, University of Chicago, Chicago, Illinois, USA. ${ }^{48}$ Departamento de Física, Pontificia Universidad Católica de Chile, Santiago, Chile. ${ }^{49}$ Departamento de Física, Universidad Técnica Federico Santa María, Valparaíso, Chile. ${ }^{50}$ Institute of High Energy Physics, Chinese Academy of Sciences, Beijing, China. ${ }^{51}$ Department of Physics, Nanjing University, Jiangsu, China. ${ }^{52}$ Physics Department, Tsinghua University, Beijing 100084, China. ${ }^{53}$ Department of Modern Physics, University of Science and Technology of China, Anhui, China. ${ }^{54}$ School of Physics, Shandong University, Shandong, China. ${ }^{55}$ Department of Physics and Astronomy, Key Laboratory for Particle Physics, Astrophysics and Cosmology, Ministry of Education, Shanghai Key Laboratory for Particle Physics and Cosmology, Shanghai Jiao Tong University, Shanghai (also at PKU-CHEP), China. ${ }^{56}$ Université Clermont Auvergne, CNRS/IN2P3, LPC, Clermont-Ferrand, France. ${ }^{57}$ Nevis Laboratory, Columbia University, Irvington, New York, USA. ${ }^{58}$ Niels Bohr Institute, University of Copenhagen, Kobenhavn, Denmark. ${ }^{59}$ INFN Gruppo Collegato di Cosenza, Laboratori Nazionali di Frascati, Rende, Italy. ${ }^{60}$ Dipartimento di Fisica, Università della Calabria, Rende, Italy. ${ }^{61} \mathrm{AGH}$ University of Science and Technology, Faculty of Physics and Applied Computer Science, Krakow, Poland. ${ }^{62}$ Marian Smoluchowski Institute of Physics, Jagiellonian University, Krakow, Poland. ${ }^{63}$ Institute of Nuclear Physics Polish Academy of Sciences, Krakow, Poland. ${ }^{64}$ Physics Department, Southern Methodist University, Dallas, Texas, USA. ${ }^{65}$ Physics Department, University of Texas at Dallas, Richardson, Texas, USA. ${ }^{66}$ DESY, Hamburg and Zeuthen, Germany. ${ }^{67}$ Lehrstuhl für Experimentelle Physik IV, Technische Universität Dortmund, Dortmund, Germany. ${ }^{68}$ Institut für Kern- und Teilchenphysik, Technische Universität Dresden, Dresden, Germany. ${ }^{69}$ Department of Physics, Duke University, Durham, North Carolina, USA. ${ }^{70}$ SUPA - School of Physics and Astronomy, University of Edinburgh, Edinburgh, UK. ${ }^{71}$ INFN Laboratori Nazionali di Frascati, Frascati, Italy. ${ }^{72}$ Fakultät für Mathematik und Physik, Albert-Ludwigs-Universität, Freiburg, Germany. ${ }^{73}$ Departement de Physique Nucleaire et Corpusculaire, Université de Genève, Geneva, Switzerland. ${ }^{74}$ INFN Sezione di Genova, Genova, Italy. ${ }^{75}$ Dipartimento di Fisica, Università di Genova, Genova, Italy. ${ }^{76}$ E. Andronikashvili Institute of Physics, Iv. Javakhishvili Tbilisi State University, Tbilisi, Georgia. ${ }^{77}$ High Energy Physics Institute, Tbilisi State University, Tbilisi, Georgia. ${ }^{78}$ II Physikalisches Institut, Justus-Liebig-Universität Giessen, Giessen, Germany. ${ }^{79}$ SUPA - School of Physics and Astronomy, University of Glasgow, Glasgow, UK. ${ }^{80}$ II Physikalisches Institut, Georg-August-Universität, Göttingen, Germany. ${ }^{81}$ Laboratoire de Physique Subatomique et de Cosmologie, Université Grenoble-Alpes, CNRS/IN2P3, Grenoble, France. ${ }^{82}$ Laboratory for Particle Physics and Cosmology, Harvard University, Cambridge, Massachusetts, USA. ${ }^{83}$ Kirchhoff-Institut für Physik, Ruprecht-Karls-Universität Heidelberg, Heidelberg, Germany. ${ }^{84}$ Physikalisches Institut, Ruprecht-Karls-Universität Heidelberg, Heidelberg, Germany. ${ }^{85}$ ZITI Institut für Technische Informatik, Ruprecht-Karls-Universität Heidelberg, Mannheim, Germany. ${ }^{86}$ Faculty of Applied Information Science, Hiroshima Institute of Technology, Hiroshima, Japan. ${ }^{87}$ Department of Physics, The Chinese University of Hong Kong, Shatin, N.T., Hong Kong, China. ${ }^{88}$ Department of Physics, The University of Hong Kong, Hong Kong, China. 
${ }^{89}$ Department of Physics and Institute for Advanced Study, The Hong Kong University of Science and Technology, Clear Water Bay, Kowloon, Hong Kong, China. ${ }^{90}$ Department of Physics, National Tsing Hua University, Taiwan, Taiwan. ${ }^{91}$ Department of Physics, Indiana University, Bloomington, Indiana, USA. ${ }^{92}$ Institut für Astro- und Teilchenphysik, Leopold-Franzens-Universität, Innsbruck, Austria. ${ }^{93}$ University of lowa, lowa City, lowa, USA. ${ }^{94}$ Department of Physics and Astronomy, lowa State University, Ames, lowa, USA. ${ }^{95}$ Joint Institute for Nuclear Research, JINR Dubna, Dubna, Russia. ${ }^{96}$ KEK, High Energy Accelerator Research Organization, Tsukuba, Japan. ${ }^{97}$ Graduate School of Science, Kobe University, Kobe, Japan. ${ }^{98}$ Faculty of Science, Kyoto University, Kyoto, Japan. ${ }^{99}$ Kyoto University of Education, Kyoto, Japan. ${ }^{100}$ Department of Physics, Kyushu University, Fukuoka, Japan. ${ }^{101}$ Instituto de Física La Plata, Universidad Nacional de La Plata and CONICET, La Plata, Argentina. ${ }^{102}$ Physics Department, Lancaster University, Lancaster, UK. ${ }^{103}$ INFN Sezione di Lecce, Lecce, Italy. ${ }^{104}$ Dipartimento di Matematica e Fisica, Università del Salento, Lecce, Italy. ${ }^{105}$ Oliver Lodge Laboratory, University of Liverpool, Liverpool, UK. ${ }^{106}$ Department of Experimental Particle Physics, Jožef Stefan Institute and Department of Physics, University of Ljubljana, Ljubljana, Slovenia. ${ }^{107}$ School of Physics and Astronomy, Queen Mary University of London, London, UK. ${ }^{108}$ Department of Physics, Royal Holloway University of London, Egham, UK. ${ }^{109}$ Department of Physics and Astronomy, University College London, London, UK. ${ }^{110}$ Louisiana Tech University, Ruston, Louisiana, USA. ${ }^{111}$ Laboratoire de Physique Nucléaire et de Hautes Energies, UPMC and Université Paris-Diderot and CNRS/IN2P3, Paris, France. ${ }^{112}$ Fysiska Institutionen, Lunds Universitet, Lund, Sweden. ${ }^{113}$ Departamento de Fisica Teorica C-15, Universidad Autonoma de Madrid, Madrid, Spain. ${ }^{114}$ Institut für Physik, Universität Mainz, Mainz, Germany. ${ }^{115}$ School of Physics and Astronomy, University of Manchester, Manchester, UK. ${ }^{116}$ CPPM, Aix-Marseille Université and CNRS/IN2P3, Marseille, France. ${ }^{117}$ Department of Physics, University of Massachusetts, Amherst, Massachusetts, USA. ${ }^{118}$ Department of Physics, McGill University, Montreal, Quebec, Canada. ${ }^{119}$ School of Physics, University of Melbourne, Victoria, Australia. ${ }^{120}$ Department of Physics, The University of Michigan, Ann Arbor, Michigan, USA. ${ }^{121}$ Department of Physics and Astronomy, Michigan State University, East Lansing, Michigan, USA. ${ }^{122}$ INFN Sezione di Milano, Milano, Italy. ${ }^{123}$ Dipartimento di Fisica, Università di Milano, Milano, Italy. ${ }^{124}$ B.I. Stepanov Institute of Physics, National Academy of Sciences of Belarus, Minsk, Republic of Belarus. ${ }^{125}$ Research Institute for Nuclear Problems of Byelorussian State University, Minsk, Republic of Belarus. ${ }^{126}$ Group of Particle Physics, University of Montreal, Montreal, Quebec, Canada. ${ }^{127}$ P.N. Lebedev Physical Institute of the Russian Academy of Sciences, Moscow, Russia. ${ }^{128}$ Institute for Theoretical and Experimental Physics (ITEP), Moscow, Russia. ${ }^{129}$ National Research Nuclear University MEPhl, Moscow, Russia. ${ }^{130}$ D.V. Skobeltsyn Institute of Nuclear Physics, M.V. Lomonosov Moscow State University, Moscow, Russia. ${ }^{131}$ Fakultät für Physik, Ludwig-Maximilians-Universität München München, Germany. ${ }^{132}$ Max-Planck-Institut für Physik (Werner-Heisenberg-Institut), München, Germany. ${ }^{133}$ Nagasaki Institute of Applied Science, Nagasaki, Japan. ${ }^{134}$ Graduate School of Science and Kobayashi-Maskawa Institute, Nagoya University, Nagoya, Japan. ${ }^{135}$ INFN Sezione di Napoli, Napoli, Italy. ${ }^{136}$ Dipartimento di Fisica, Università di Napoli, Napoli, Italy. ${ }^{137}$ Department of Physics and Astronomy, University of New Mexico, Albuquerque, New Mexico, USA. ${ }^{138}$ Institute for Mathematics, Astrophysics and Particle Physics, Radboud University Nijmegen/Nikhef, Nijmegen, the Netherlands. ${ }^{139}$ Nikhef National Institute for Subatomic Physics and University of Amsterdam, Amsterdam, the Netherlands. ${ }^{140}$ Department of Physics, Northern Illinois University, DeKalb, Illinois, USA. ${ }^{141}$ Budker Institute of Nuclear Physics, SB RAS, Novosibirsk, Russia. ${ }^{142}$ Department of Physics, New York University, New York, New York, USA. ${ }^{143}$ Ohio State University, Columbus, Ohio, USA. ${ }^{144}$ Faculty of Science, Okayama University, Okayama, Japan. ${ }^{145}$ Homer L. Dodge Department of Physics and Astronomy, University of Oklahoma, Norman, Oklahoma, USA. ${ }^{146}$ Department of Physics, Oklahoma State University, Stillwater, Oklahoma, USA. ${ }^{147}$ Palacký University, RCPTM, Olomouc, Czech Republic. ${ }^{148}$ Center for High Energy Physics, University of Oregon, Eugene, Oregon, USA. ${ }^{149}$ LAL, Univ. Paris-Sud, CNRS/IN2P3, Université Paris-Saclay, Orsay, France. ${ }^{150}$ Graduate School of Science, Osaka University, Osaka, Japan. ${ }^{151}$ Department of Physics, University of Oslo, Oslo, Norway. ${ }^{152}$ Department of Physics, Oxford University, Oxford, UK. ${ }^{153}$ INFN Sezione di Pavia, Pavia, Italy. ${ }^{154}$ Dipartimento di Fisica, Università di Pavia, Pavia, Italy. ${ }^{155}$ Department of Physics, University of Pennsylvania, Philadelphia, Pennsylvania, USA.

${ }^{156}$ National Research Centre "Kurchatov Institute" B.P. Konstantinov Petersburg Nuclear Physics Institute, St Petersburg, Russia. ${ }^{157}$ INFN Sezione di Pisa, Pisa, Italy. ${ }^{158}$ Dipartimento di Fisica E. Fermi, Università di Pisa, Pisa, Italy. ${ }^{159}$ Department of Physics and Astronomy, University of Pittsburgh, Pittsburgh, Pennsylvania, USA. ${ }^{160}$ Laboratório de Instrumentação e Física Experimental de Partículas - LIP, Lisboa, Portugal. ${ }^{161}$ Faculdade de Ciências, Universidade de Lisboa, Lisboa, Portugal. ${ }^{162}$ Department of Physics, University of Coimbra, Coimbra, Portugal. ${ }^{163}$ Centro de Física Nuclear da Universidade de Lisboa, Lisboa, Portugal. ${ }^{164}$ Departamento de Fisica, Universidade do Minho, Braga, Portugal. ${ }^{165}$ Departamento de Fisica Teorica y del Cosmos and CAFPE, Universidad de Granada, Granada (Spain), Portugal. ${ }^{166}$ Dep Fisica and CEFITEC of Faculdade de Ciencias e Tecnologia, Universidade Nova de Lisboa, Caparica, Portugal. ${ }^{167}$ Institute of Physics, Academy of Sciences of the Czech Republic, Praha, Czech Republic. ${ }^{168}$ Czech Technical University in Prague, Praha, Czech Republic. ${ }^{169}$ Charles University, Faculty of Mathematics and Physics, Prague, Czech Republic. ${ }^{170}$ State Research Center Institute for High Energy Physics (Protvino), NRC KI, Russia. ${ }^{171}$ Particle Physics Department, Rutherford Appleton Laboratory, Didcot, UK. ${ }^{172}$ INFN Sezione di Roma, Roma, Italy. ${ }^{173}$ Dipartimento di Fisica, Sapienza Università di Roma, Roma, Italy. ${ }^{174}$ INFN Sezione di Roma Tor Vergata, Roma, Italy. ${ }^{175}$ Dipartimento di Fisica, Università di Roma Tor Vergata, Roma, Italy. ${ }^{176}$ INFN Sezione di Roma Tre, Roma, Italy. ${ }^{177}$ Dipartimento di Matematica e Fisica, Università Roma Tre, Roma, Italy. ${ }^{178}$ Faculté des Sciences Ain Chock, Réseau Universitaire de Physique des Hautes Energies - Université Hassan II, Casablanca, Morocco. ${ }^{179}$ Centre National de l'Energie des Sciences Techniques Nucleaires, Rabat, Morocco. ${ }^{180}$ Faculté des Sciences Semlalia, Université Cadi Ayyad, LPHEA-Marrakech, Morocco. ${ }^{181}$ Faculté des Sciences, Université Mohamed Premier and LPTPM, Oujda, Morocco. ${ }^{182}$ Faculté des sciences, Université Mohammed V, Rabat, Morocco. ${ }^{183} \mathrm{DSM}$ /IRFU (Institut de Recherches sur les Lois Fondamentales de I'Univers), CEA Saclay (Commissariat à I'Energie Atomique et aux Energies Alternatives), Gif-sur-Yvette, France. ${ }^{184}$ Santa Cruz Institute for Particle Physics, University of California Santa Cruz, Santa Cruz, California, USA. ${ }^{185}$ Department of Physics, University of Washington, Seattle, Washington, USA. ${ }^{186}$ Department of Physics and Astronomy, University of Sheffield, Sheffield, UK. ${ }^{187}$ Department of Physics, Shinshu University, Nagano, Japan. ${ }^{188}$ Department Physik, Universität Siegen, Siegen, Germany. ${ }^{189}$ Department of Physics, Simon Fraser University, Burnaby, British Columbia, Canada. ${ }^{190}$ SLAC National Accelerator Laboratory, Stanford, California, USA. ${ }^{191}$ Faculty of Mathematics, Physics \& Informatics, Comenius University, Bratislava, Slovak Republic. ${ }^{192}$ Department of Subnuclear Physics, Institute of Experimental Physics of the Slovak Academy of Sciences, Kosice, Slovak Republic. ${ }^{193}$ Department of Physics, University of Cape Town, Cape Town, South Africa. ${ }^{194}$ Department of Physics, University of Johannesburg, Johannesburg, South Africa. ${ }^{195}$ School of Physics, University of the Witwatersrand, Johannesburg, South Africa. ${ }^{196}$ Department of Physics, Stockholm University, Stockholm, Sweden. ${ }^{197}$ The Oskar Klein Centre, Stockholm, Sweden. ${ }^{198}$ Physics Department, Royal Institute of Technology, Stockholm, Sweden. ${ }^{199}$ Departments of Physics \& Astronomy and Chemistry, Stony Brook University, Stony Brook, New York, USA. ${ }^{200}$ Department of Physics and Astronomy, University of Sussex, Brighton, UK. ${ }^{201}$ School of Physics, University of Sydney, Sydney, Australia. 202 Institute of Physics, Academia Sinica, Taipei, Taiwan. ${ }^{203}$ Department of Physics, Technion: Israel Institute of Technology, Haifa, Israel. 204 Raymond and Beverly Sackler School of Physics and Astronomy, Tel Aviv University, Tel Aviv, Israel. ${ }^{205}$ Department of Physics, Aristotle University of Thessaloniki, Thessaloniki, Greece. ${ }^{206}$ International Center for Elementary Particle Physics and Department of Physics, The University of Tokyo, Tokyo, Japan. ${ }^{207}$ Graduate School of Science and Technology, Tokyo Metropolitan University, Tokyo, Japan. ${ }^{208}$ Department of Physics, Tokyo Institute of Technology, Tokyo, Japan. ${ }^{209}$ Tomsk State University, Tomsk, Russia. ${ }^{210}$ Department of Physics, University of Toronto, Toronto, Ontario, Canada. ${ }^{211}$ INFN-TIFPA, Trento, Italy. ${ }^{212}$ University of Trento, Trento, Italy. ${ }^{213}$ TRIUMF, Vancouver, British Columbia, Canada. ${ }^{214}$ Department of Physics and Astronomy, York University, Toronto, Ontario, Canada. ${ }^{215}$ Faculty of Pure and Applied Sciences, and Center for Integrated Research in Fundamental Science and Engineering, University of Tsukuba, Tsukuba, Japan. ${ }^{216}$ Department of Physics and Astronomy, Tufts University, Medford, Massachusetts, USA. ${ }^{217}$ Department of Physics and Astronomy, University of California Irvine, Irvine, California, USA. ${ }^{218}$ INFN Gruppo Collegato di Udine, Sezione di Trieste, Udine, Italy. ${ }^{219}$ ICTP, Trieste, Italy. ${ }^{220}$ Dipartimento di Chimica, Fisica e Ambiente, Università di Udine, Udine, Italy. ${ }^{221}$ Department of Physics and Astronomy, University of Uppsala, Uppsala, Sweden. ${ }^{222}$ Department of Physics, University of Illinois, Urbana, Illinois, USA. ${ }^{223}$ Instituto de Fisica Corpuscular (IFIC) and Departamento de Fisica Atomica, Molecular y Nuclear and Departamento de Ingeniería Electrónica and Instituto de Microelectrónica de Barcelona (IMB-CNM), University of Valencia and CSIC, Valencia, Spain. ${ }^{224}$ Department of Physics, University of British Columbia, Vancouver, British Columbia, Canada. ${ }^{225}$ Department of 
Physics and Astronomy, University of Victoria, Victoria, British Columbia, Canada. ${ }^{226}$ Department of Physics, University of Warwick, Coventry, UK.

${ }^{227}$ Waseda University, Tokyo, Japan. ${ }^{228}$ Department of Particle Physics, The Weizmann Institute of Science, Rehovot, Israel. ${ }^{229}$ Department of Physics, University of Wisconsin, Madison, Wisconsin, USA. ${ }^{230}$ Fakultät für Physik und Astronomie, Julius-Maximilians-Universität, Würzburg, Germany.

${ }^{231}$ Fakultät für Mathematik und Naturwissenschaften, Fachgruppe Physik, Bergische Universität Wuppertal, Wuppertal, Germany. ${ }^{232}$ Department of Physics, Yale University, New Haven, Connecticut, USA. ${ }^{233}$ Yerevan Physics Institute, Yerevan, Armenia. ${ }^{234} \mathrm{CH}-1211$, Geneva 23 , Switzerland. ${ }^{235} \mathrm{Centre}$ de Calcul de I'Institut National de Physique Nucléaire et de Physique des Particules (IN2P3), Villeurbanne, France.

\section{Secondary affiliations}

${ }^{a}$ Department of Physics, King's College London, London, UK. 'b Institute of Physics, Azerbaijan Academy of Sciences, Baku, Azerbaijan. ${ }^{\mathrm{c} N o v o s i b i r s k ~ S t a t e}$ University, Novosibirsk, Russia. ${ }^{\mathrm{d}}$ TRIUMF, Vancouver, British Columbia, Canada. ${ }^{e}$ Department of Physics \& Astronomy, University of Louisville, Louisville, Kentucky, USA. ${ }^{f}$ Physics Department, An-Najah National University, Nablus, Palestine. ${ }^{8}$ Department of Physics, California State University, Fresno, California, USA. ' Department of Physics, University of Fribourg, Fribourg, Switzerland. 'Il Physikalisches Institut, Georg-August-Universität, Göttingen, Germany. 'Departament de Fisica de la Universitat Autonoma de Barcelona, Barcelona, Spain. ${ }^{k}$ Departamento de Fisica e Astronomia, Faculdade de Ciencias, Universidade do Porto, Portugal. 'Tomsk State University, Tomsk, Russia. 'm The Collaborative Innovation Center of Quantum Matter (CICQM),

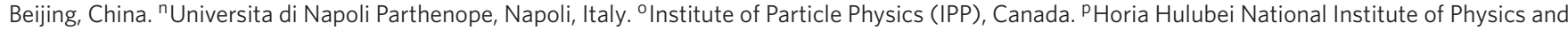
Nuclear Engineering, Bucharest, Romania. ${ }^{9}$ Department of Physics, St Petersburg State Polytechnical University, St Petersburg, Russia. ' ${ }^{\text {Borough of }}$ Manhattan Community College, City University of New York, New York City, USA. 'Department of Physics, The University of Michigan, Ann Arbor, Michigan, USA. ${ }^{\mathrm{t} C e n t r e ~ f o r ~ H i g h ~ P e r f o r m a n c e ~ C o m p u t i n g, ~ C S I R ~ C a m p u s, ~ R o s e b a n k, ~ C a p e ~ T o w n, ~ S o u t h ~ A f r i c a . ~ " ~ L o u i s i a n a ~ T e c h ~ U n i v e r s i t y, ~ R u s t o n, ~}$ Louisiana, USA. v'Institucio Catalana de Recerca i Estudis Avancats, ICREA, Barcelona, Spain. wGraduate School of Science, Osaka University, Osaka, Japan. ${ }^{x}$ Fakultät für Mathematik und Physik, Albert-Ludwigs-Universität, Freiburg, Germany. ${ }^{\mathrm{Y}}$ Institute for Mathematics, Astrophysics and Particle Physics, Radboud University Nijmegen/Nikhef, Nijmegen, the Netherlands. ${ }^{2}$ Department of Physics, The University of Texas at Austin, Austin, Texas, USA. aa Institute of Theoretical Physics, Ilia State University, Tbilisi, Georgia. ab CERN, Geneva, Switzerland. ac Georgian Technical University (GTU), Tbilisi, Georgia. ${ }^{\text {ad } O c h a d a i ~ A c a d e m i c ~ P r o d u c t i o n, ~ O c h a n o m i z u ~ U n i v e r s i t y, ~ T o k y o, ~ J a p a n . ~ a e ~ M a n h a t t a n ~ C o l l e g e, ~ N e w ~ Y o r k, ~ N e w ~ Y o r k, ~ U S A . ~ a f ~ A c a d e m i a ~ S i n i c a ~ G r i d ~}$ Computing, Institute of Physics, Academia Sinica, Taipei, Taiwan. ag School of Physics, Shandong University, Shandong, China. ah Departamento de Fisica Teorica y del Cosmos and CAFPE, Universidad de Granada, Granada (Spain), Portugal. ai Department of Physics, California State University, Sacramento, California, USA. aj Moscow Institute of Physics and Technology State University, Dolgoprudny, Russia. ak Departement de Physique Nucleaire et

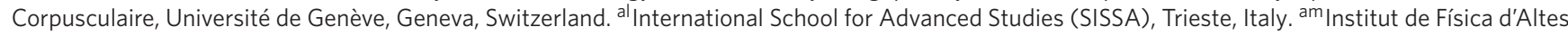
Energies (IFAE), The Barcelona Institute of Science and Technology, Barcelona, Spain. ${ }^{\text {an }}$ School of Physics, Sun Yat-sen University, Guangzhou, China. ${ }^{a}$ Institute for Nuclear Research and Nuclear Energy (INRNE) of the Bulgarian Academy of Sciences, Sofia, Bulgaria. ap Faculty of Physics, M.V. Lomonosov

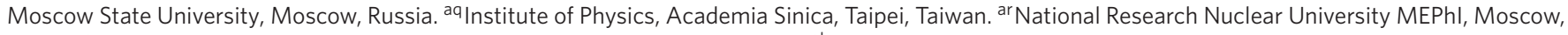
Russia. as Department of Physics, Stanford University, Stanford, California, USA. at Institute for Particle and Nuclear Physics, Wigner Research Centre for

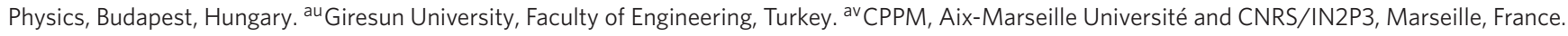
${ }^{a w}$ Department of Physics, Nanjing University, Jiangsu, China. ${ }^{a x}$ University of Malaya, Department of Physics, Kuala Lumpur, Malaysia. ay LAL, Univ. Paris-Sud, CNRS/IN2P3, Université Paris-Saclay, Orsay, France. *Deceased. *e-mail: atlas.publications@cern.ch 